\title{
LES CAHIERS D'ETUDE DE KARL MARX
}

$$
\text { II. } 1853-1856^{1}
$$

Nota bene: l'article comporte quatre chapitres et une conclusion:

- Histoire des civilisations (p. 39)

- La question d'Orient (p. 43)

- Les révolutions d'Espagne (p. 49)

- Histoire diplomatique (p. 59)

- Conclusion (p. 74)

\section{Sigles :}

BIBL. = M. Rubel. Bibliographie des Oeuvres de Karl Marx. En appendice: Répertoire des oeuvres de F. Engels. Paris, M. Rivière I956.

GR = Karl Marx. Grundrisse der Kritik der politischen Oekonomie (Rohentwurf) I 857 -1 8 58. Berlin, Dietz 1953.

GS $=$ Gesammelte Schriften von Karl Marx und Friedrich Engels. Herausgegeben von N. Rjasanoff. Zweite Auflage. Stuttgart, Dietz 1920. 2 volumes.

NY'T $=$ New York Daily Tribune.

REV. = Revolution in Spain. By K. Marx \& F. Engels. InterSPAIN national Publishers, New York 1939.

\section{HISTOIRE DES CIVILISATIONS}

Marx a lu pendant près de sept ans les ouvrages des économistes et en a fait des extraits, souvent annotés, qui emplissent une quarantaine de cahiers. En I 85 I, il se croit, avec quelque apparence de raison, au bout de ses recherches, et s'apprête à rédiger l'oeuvre méditée. L'accueil des éditeurs allemands ne l'y encourage guère. Malade, sans ressources, il se voit offrir la correspondance européenne d'un grand quotidien new-yorkais et accepte avec empressement. Le pro-

\footnotetext{
${ }^{1}$ Voir cette revue, vol. 2, 1957, no 3, p. 392-420, la première partic. N.B. - Les cotes B qui correspondent au classement des manuscrits à l'I.I.H.S. étant sujettes à révision, nous ne les indiquons qu'à titre provisoire. Dans le déchiffrement de plusieurs cahiers présentés dans le présent essai, j'ai été beaucoup aidé par Louis Evrard, à qui j'exprime ici ma reconnaissante amitié.
} 
blème est d'écrire en anglais. Il aura recours à Engels et signera les articles que son ami lui enverra pendant plusieurs mois de Manchester. ${ }^{1}$

A partir de 1852, Marx écrira lui-même ses articles. En allemand d'abord, se faisant traduire par un ami de Londres; puis en anglais, sans cependant renoncer au concours d'Engels.

De 1853 à 1857 , c'est le journalisme qui sera la principale occupation de Marx. ${ }^{2}$

En 1857 - 1858 , il pourra procéder à une première rédaction de son oeuvre scientifique, et il publiera en 1859 le premier fascicule de Zur Kritik der politischen OKkonomie. Les événements politiques de 1859-1860 le détournent de cette voie, et ce n'est qu'à partir de I86I qu'il pourra reprendre, sous une nouvelle forme, la rédaction du Capital.

Ainsi, de 1853 à I 860 , Marx a publié près de cinq cents articles en anglais ou en allemand, plusieurs brochures et deux ouvrages; ${ }^{3}$ de cette période date également le grand manuscrit des Grundrisse que nous ne connaissons que depuis I94I.

Pour les articles, les cahiers de lecture apparaissent comme des réserves de matériaux prêts à servir au commentaire des événements européens.

Marx n'avait rien d'un correspondant au sens ordinaire du terme, rien d'un compilateur. ${ }^{4}$ Ses premiers articles, consacrés aux élections anglaises de juillet I 852 et envoyés à la NYT avec un certain retard, sont d'un historien plutôt que d'un journaliste. C'est encore plus vrai de ses articles sur la question d'Orient, revenue à l'actualité au printemps 1853.5

Plusieurs cahiers ont un lien direct avec les articles publiés par Marx dans la NYT la même année et en i 854 . Nous avons déjà parlé du cahier daté de janvier 1853 , contenant des extraits de quelques importants ouvrages sur l'histoire de la civilisation et sur la théorie de la population. ${ }^{6}$ Herbert Spencer, G. Opdyke, F. W. Newman et T. C.

1 Voir leur correspondance d'août et septembre I 85 I.

2 Voici les journaux auxquels Marx a donné des contributions importantes: NYT (18 5 2I862); Neue Oder-Zeitung (1855); Free Press (1856-1859); Das Volk (1859); Die Presse (1861-1862).

${ }^{3}$ Enthüllungen über den Kommunistenprozess zu Köln, 1853. Der Ritter von edelmütigen Bewusstsein, 1853. Zur Kritik der politischen Okonomie/Erstes Heft, 1959. Herr Vogt, I 86o. On peut également mentionner le manuscrit „commandé” par Bangya Die grossen Männer des Exils (BIBL. 2 I 7 P) dont seul un brouillon est conservé.

4 Voir Riazanov, introduction à GS I, p. XXVI.

${ }^{5}$ Il serait toutefois faux de penser que le journalisme, même sérieux, eût satisfait Marx intellectuellement, bien au contraire: voir sa lettre à E., 23.1.1857.

Article précédent, p. 4I4. Voir l'article dans la NYT, 22.3.1853 (pression démographique et migrations antiques et modernes). 
Banfield y figurent avec de nombreux passages qui débordent le cadre de l'économie politique; ces auteurs semblent avoir été consultés par Marx avec une curiosité que l'on pourrait dire sociologique. Ainsi par exemple, le passage suivant, tiré de H. Spencer, Social Statics... (18 5 1), p. 442, y est fortement souligné:

«...When civilization becomes complete, will the normal unfolding of all other elements of the individuality depend upon the existence of the civilized state. Just that kind of individuality will be acquired which finds in the most highly organized community the fittest sphere for its manifestation [passage sauté par Marx: which finds in each social arrangement a condition answering to some faculty in itself which could not, in fact, expand at all, of otherwise circumstanced]. The ultimate man will be one whose private requirements coincide with public ones. He will be that manner of man, who, in spontaneously fulfilling his own nature, incidentally performs the functions of a social unit; and yet is only enabled so to fulfil his own nature, by all others doing the like. ${ }^{1}$

L'histoire de l'Orient et plus particulièrement de l'Inde occupe dans ce même cahier une place importante. On en comprend la raison: la question d'Orient était une fois de plus au centre de l'actualité européenne, et Marx ne pouvait pas se laisser distancer par ses rivaux qui, du continent et d'Angleterre, envoyaient leurs informations aux journaux américains. ${ }^{2}$ D'autre part, la Grande-Bretagne était en difficulté aux Indes Orientales; et l'on pouvait prévoir que la Russie ne laisserait pas échapper une occasion de pousser son expansion vers l'Asie.

Une trentaine de pages du cahier (qui en compte soixante-dix) contiennent une ample moisson de renseignements et considérations sur l'Inde, glanés chez des auteurs et dans des documents divers. ${ }^{3} \mathrm{Y}$ figurent: Wachsmuth, Europäische Sittengeschicbte («continuation du cahier XX");4 MacCulloch, East India (in: Literature of Political Economy, London 1845); Gustav Klein, Allgemeine Culturgeschichte der Menschbeit, vol. 6(Chine) et vol. 7(Orient), Leipzig, 1847-1849; Indian Railways and their probable results... By an old Postmaster, London 1848; Lettre sur l'Inde, par le Prince Saltykow, Paris, 1849;

1 Vérifié par nous sur l'édition de Londres, 1902, p. 256.

- Les premières correspondances furent rédigées par Engels à qui Marx transmit quelques directives générales. Voir M. ̀̀ E., 10.3.1853.

- Sur les matériaux de Marx concernant l'Inde, voir L. A. Alekseiva, Vypiski K. Marksa po istorii Indii: dans: Problemy Vistokevedeniia, 1959, no 2, p. I63-9; d'après les photocopies des cahiers, en possession de l'Institut du Marxisme-Léninisme.

- Voir article précédent, p. 413. 
MacCulloch, East India (in: A Dictionary, practical, theoretical and historical of Commerce and commercial navigation, London I847); Observations on India, By a Resident there many years, London I 853 . D'un écrit anonyme, India, Great Britain and Russia, London, 1838 , Marx a noté un passage dont voici le début: "Beyond Persia lies Afghanistan.... The Indus crossed, what is to resist the flight of the Russian eagle into the heart of british India?"

Dans l'ouvrage de A. H. Heeren, De la politique et du commerce des peuples de l'antiquité (trad. par W. Suckau), Paris 1833 (t. III), Marx a copié une vingtaine de passages se rapportant à la culture architecturale, aux coutumes religieuses, aux castes, aux institutions primitives, à la législation, etc. de l'Inde.

Plus nombreux encore sont les extraits notés par Marx de François Bernier, Voyages contenant la description des Etats du Grand Mogol, de l'Hindoustan, etc., Amsterdam, 1799 (réédité à Paris 1830). On connaît l'éloge que Marx fit de cet ouvrage dans une lettre à Engels. ${ }^{1}$

De juin à septembre 1853, Marx écrivit plusieurs articles sur la politique anglaise dans l'Inde. ${ }^{2} \mathrm{La}$ haute tenue de ces correspondances fait supposer de bonnes lectures. En fait, deux cahiers, datés de juin I 853 et portant les chiffres «XXII», et «XXIII» nous renseignent sur les sources utilisées par Marx non seulement pour ces articles, mais aussi pour son oeuvre maîtresse. ${ }^{3}$

Voici les principaux titres du cahier XXII:

Th. Stamford Raffles, The History of Java. London, Black, P. \& A., I 817,2 vol. ${ }^{4}$

Lieut.-Col. Mark Wilks, Historical Sketches of the South of India, in an attempt to trace the bistory of Mysore, 3 vol., London, $1810-1817 .{ }^{5}$

H. Murray, J. Wilson, Greville, etc., Historical and descriptive Account of British India from the most remote periode to the present time, 3 vol. Edinburgh, I832.

George Campbell, Modern India, A Sketch of the System of Civil Government. London I $852 .^{6}$ Marx en a copié des extraits sur plus de dix-huit pages.

Les extraits se rapportent principalement à l'histoire et à la civilisation

1 M. à E., 2.6.1853, E. à M., 6.6.1853.

${ }^{2}$ Voir BIBL. 252, 253, 254, 256, 258,260, 262, 263 auxquels il convient d'ajouter 256 NYT, II.7.1853 et $274^{2}$, NYT. 4.10.1853. Sur 253 (The British Rule in India, NYT, 25.6.1853) voir Marx à Engels, I 4 juin I 853 . Marx y cite un rapport parlementaire sur les villages hindous.

${ }^{3}$ Classés actuellement sous B 66 et B 65 .

${ }^{4}$ Cité par Marx dans sa lettre à Engels du 14.6.1853 et dans Das Kapital, I, chap. I 2.

${ }^{5}$ Cité dans Das Kapital I, chap. I 2.

${ }^{6}$ Cité dans Das Kapital I, chap. 12. Le cahier, folio ro, donne le titre comme suit: „Bengal Civil Service. Modern India". London 1852. 
de l'Inde jusqu'au déclin de l'Empire Mongol, extraits souvent purement chronologiques; aux classes et castes, à la densité de la population, au revenu de la terre, aux diverses sources de revenus, etc. Il faut signaler les considérations sur la constitution des villages avec des allusions comparatives aux marches germaniques et villages celtes, etc.

Du même auteur, nous trouvons dans ce cahier quelques passages d'un texte intitule $A$ Schema for the Government of India, London 1853. Ils sont suivis d'extraits de Robert Patton, The Principles of Asiatic Monarcby, London I 801 .

Au cahier XXIII nous trouvons sous le titre «Schriften publiciert durch d. India Reform Association" (London 1853 ), douze pages de notes, d'après les sept brochures suivantes: I. The Government of India since ${ }_{1} 834$. II. The Finances of India... III. Notes on India... IV. The Native States of India. V. Extract from Mill's bistory on the double government... by John Sullivan... VI. John Dickinson, The Government in India under a Bureaucracy VII. India Wrongs witbout a Remedy.

\section{LA QUESTION D'ORIENT}

C'est sur la crise d'Orient que Marx écrit pour la première fois sans l'aide d'Engels. ${ }^{1}$ On en trouve un premier essai dans un article paru dans la NYT du 9.6.1853, traitant entre autres de la Russie et de la Turquie. ${ }^{2}$ Marx lit alors plusieurs documents sur lesquels le cahier XXII nous renseigne:

The Portfolio de 1843 (fondé par D. Urquhart) dont Marx a cité, dans un article, le télégramme adressé par le prince Lieven à Nesselrode, le I(I 3).I0.I 829, prouvant les sentiments russophiles de lord Aberdeen. ${ }^{3}$ J. Ph. Fallmerayer, Fragmente aus dem Oriente, I845. Les passages recopiés par Marx concernent l'histoire des peuples slaves. ${ }^{4}$

En juin r 853, Marx se propose d'écrire sur la question du SlesvigHolstein croyant y déceler, comme dans la question d'Orient, «le principe de l'expansionnisme de la Russie. ${ }^{5}$ Le cahier XXII contient quelques matériaux sur cette question:

\footnotetext{
${ }^{1}$ A propos de la "détestable question orientale", voir Marx à Engels, 10.3.1853. Le 22 mars, Marx transmet à Engels des informations, tirées de l'Economist, sur les exportations anglaises vers la Russie et la Turquie. Engels utilisa ces chiffres dans une correspondance envoyée à la NYT où elle fut publiée comme éditorial, le I 2.4.1853. Cf. GS I, p. I 54 sq. GS I, p. r 70 sq.

${ }^{3}$ Paru dans NYT, I4.7.1853. GS I, p. 183 sq. et 478 sq. (importance du Portfolio comme source de Marx).

- Sur l'influence des idées de Fallmerayer chez M. et E., voir Riazanov, GS, I, p. 471 .

- Il avait effleuré cette question dans une correspondance parue dans la NYT, 9.6.1853. Le 30.9.1853, il informe Engels qu'il écrira trois articles sur la question danoise, mais ne semble pas avoir donné suite à ce projet.
} 
Des extraits de l'article Dänemark dans l'Encyclopédie Universelle de J. S. Ersch et de J. G. Gruber, Leipzig, I818-1850.

Une page et demie d'extraits de: Ueber das Wabre Verbaeltnis des Herzogs von Augustenburg zum Holsteinischen Aufrubre, Copenhagen 1849 , de C.-F. Wegener.

Deux pages d'extraits de Die Herrogtümer Scbleswig-Holstein und das Königreich Dänemark (1 850 ) par J. G. Droysen et H. Samwer.

Deux pages d'extraits de Das dänische Königsgesetz...(traduction), Kiel I838, par Theodor Olshausen.

Un cahier sans date, contenant vingt pages d'extraits semble appartenir à la même période qu'un autre daté de septembre 1853 , petit $8^{0}$, avec quatre-vingts pages d'extraits. ${ }^{1}$ L'un et l'autre contiennent des matériaux documentaires dont Marx s'est servi pour ses articles de la NYT et People's Paper sur la crise d'Orient, la guerre russo-turque et sur Palmerston, d'août à fin 1853 (peut-être aussi sur la guerre de Crimée).

En marge du folio I du premier, on lit cette épigraphe: «Je hais ceux qui me résistent. Je réprouve ceux qui me suivent. "

Les documents diplomatiques consultés par Marx portent sur la politique extérieure de Palmerston lors de la crise de 1840 . Du livre de D. Urquhart, La Crise (Paris I 840), Marx note entre autres, à propos du traité de la Quadruple Alliance: «La France arme, et se prépare à la guerre avec une hâte et sur une grande échelle dont son histoire n'offre pas d'exemple; mais elle ne dit rien au sujet du traité contre lequel elle prépare ses armements... elle n'en a pas exigé l'annulation.... Du même Urquhart: An appeal against faction, London 1843.

Le Portfolio de I 843 est largement exploité pour l'histoire de la diplomatie russe depuis I829, la question serbe, la guerre des Lieux saints, l'Irlande, etc. Citons en passant, pour son intérêt psychologique, un extrait (probablement d'une lettre) qui se range sous le titre: Effect of words on the cbaracter of man, and the fate of nations. Ces lignes remarquables traitent de l'opération mentale des mots et de l'aliénation par les mots:

"A new habit has been introduced amongst the nations of modern Europe, that of converting into a phantom whatever they see, and this they call 'accounting' for things... As soon as accounted for it is discarded as worthless. This is done by the simple process of uttering general propositions... When the habit of uttering general propositions, including in them the name of fanciful beings, has taken possession of a man his thought become the mere domain of [chance?], his conclusions necessarily erroneous, and his brain is left at the mercy of the accidental movements of

${ }^{1}$ Le premier est classé actuellement sous B 67 , le second sous B 63 . 
his lips... By such vain words ([like?] civilization and barbarism) are facts destroyed, and all living agency effaced in human events, and men and nature, accustomed they [are?] to use their tongues, look on their own acts as changes of the seasons, fixed by laws above their controls... endeavour to clamber by words out of the sphere of words... mist saised by the breath which abuses the mind of its sight... At each period the terms in use are accepted by the child that learns to speak before a conscious mental operation is performed, and after terms have become habitual in him no further mental operation is in his power, except [by?] flowing from and dependent upon such terms... It is speech that constitutes a nation - it is the [fabric?] of its corporate existence - it is the stream of its existence, and [links?] together in its change the first and latter days of a race, itself effecting that change, leading them greatness, or bringing them to death... In the age of activity and progress, when the cause of their greatness were chiefly in operation, did any one of these people explain what he was or endeavour to interpret the cause at his advancement? No words are only used after the thing has expired - after the spirit has itself declined. The classical age is the age preceding decay (when words came in) - the symptom and the aggravation of the disease. Man can know only what he is, and he observes what he is only by comparison with dissimilar things. The character of a nation is universal, otherwise it would not be that of the nation, and being universal it cannot be observed... A nation can recover only by removing the cause of decay. General propositions... in the fictitious representations of community you have lost man... The Greek or the Roman could personify as well as the Englishman: but he did not substitute the shadow for the reality. His image was for the imagination only... It was not an artificial being let loose on his fancy, and [poured?] forth in his language, which he invested with the power of acting on him and for him... the phantom state... I am a shadow; I am absorbed. I belong to a non-entity, to a principle. I am a whig, or a tory, or a liberal, or a conservative. He does not say I see or do - but I am - something else than self - that something being a manner of seeing, thinking, acting. *

Pour ses articles sur la question d'Orient, Marx a beaucoup puisé dans César Famin, Histoire de la rivalité et du protectorat des églises cbrétiennes en Orient, Paris 1853 . Les extraits de ce travail concernent les rapports des nations chrétiennes avec la Porte ottomane, l'origine et la nature des capitulations. Marx a recopié en particulier les articles 
I, 29, 32, 35, 82, 83, 84, etc. du traité entre la France et la Porte. Des notes chronologiques et des chifffres sur les nationalités et les fidèles, des passages sur les Lieux saints, les Croisades, etc.

Intercalés dans les extraits de Famin, quelques titres notés en vue d'articles sur les rapports de Kossuth et de Mazzini: ${ }^{1}$ Jules de Bréval: Mazzini, jugé par lui-mème et les siens (Paris 1853).

V. Gioberti: Operette politiche.

F. D. Guerrazzi: Apologia (Florence, I 8 5 I).

Massimo T. d'Azeglio: Scritti politici.

F. A. Gualterio: Gli ultimi rivolgimenti italiani...(Florence, $1850-185 \mathrm{I}$ )

L. C. Farini: Lo Stato romano (dall'anno i 8 is al i $850,2 \mathrm{ed}$. Florence, I $850-1851$ ).

N. Bianchi: Mazzini e le sue utopie... (1854?).

Et ces notations:

«Dandolo, un des chefs de l'insurrection de Milan de 1848 , un des combattants du siège de Rome 'Quest'uomo guasta tutto ciò che tocca' (Garibaldi)... ribombo du genre Mazzini... un Masanièllo de rhétorique..."

V. Gioberti (Rinnovamento) Plagiat de Mahomet: «dio è dio e l'umanita è suo profeta. ${ }^{2}$

Avant d'en terminer avec ce cahier, signalons au passage que les deux derniers feuillets portent des classifications sur la respiration et l'appareil labial.

$$
* *
$$

Le cahier daté de septembre 1853 rejoint le précédent par le contenu des matériaux recopiés qui se rapportent aux mêmes sujets: question d'Orient, Russie, Palmerston. ${ }^{3}$

Plus de deux pages d'extraits d'un opuscule anonyme: The Russians in Moldavia and Wallacbia ( «si viene di bursa a gran fortuna più con la frauda che con la forza, Machiavelli»), ze éd., London 1849. Des observations sur la langue roumaine «a sort of Oriental Italian", des notes chronologiques et des articles de traités recopiés, de 1393 à I 848 .

Une page d'extraits d'un document antirusse anonyme: Russlands Politik und Heere in den letzten Jabren, London 1852 . A côté d'extraits chronologiques, ce passage recopié: «Napoleon nannte den Mob von Paris de plus séditieux et rebelle», den von Wien 〈le plus bête` und den von Berlin ule plus infâme et lâchè».

${ }^{1}$ De septembre I 852 à mars I $853, \mathrm{M}$. donne à la NYT plusieurs articles polémiques contre Mazzini.

2 Il s'agit de Vincenzo Gioberti: Del Rinnovamento civile d'Italia... Parigi e Torino, G. Bocca, 185 I, 2 vol.

${ }^{3}$ Classé actuellement sous B 63 . 
Huit pages d'extraits d'un auteur anonyme: The Greek and Eastern Churches. Their History, Faith and Worship. London 1852. Plusieurs pages d'extraits des trois livres ci-après:

Rev. J. M. Neale, Introduction to the History of the Holy Eastern Cburches, London 1852.

Count Valerian Krasinski, Lectures on the Religious History of the Slavonic Nations. London 1 849. Marx cite d'après la 2e édition sous le titre: Sketch of the Religious History of the Slavonic Nations. Edinburgh I $8 \mathrm{~g}$ i. Le clergé russe, caste séparée. Difficultés de l'Eglise grecque de Pologne en 1839: conversions par la force et déportation des prêtres en Sibérie; la révision des livres sacrés altérés par la pratique ignorante, au XVIIe siècle; la secte des Raskolniks: «It is strange to find amongst the illiterate peasantry of Russia religious opinions of such a spiritual character as those held by the Malakanes... to meet amongst that peasantry doctrines which were entertained by the Gnostics who belonged to the most intellectual classes of the Roman society... Such is the case of the Doukbobors or Combattants in spirit (285) Martinists (by means of masonic Lodges)..."

Die Staatskirche Russlands im J. 1739. Nach d. russischen Synodalbericht dargestellt von einem Priester aus d. Congregation d. Oratorians. Schaff hausen I 844. Mêmes sujets que dans les extraits précédents, avec des aperçus sur les communautés protestantes et catholiques, et la notation d'un titre: Persécution et souffrances de l'église catholique en Russie, par un ancien conseiller d'Etat de Russie, Paris I 842.

Passages des traités de Koutchouk Kaïnardji (10.7.1774) et d'Andrinople (14.9.1829).

Marx a cité longuement ces clauses dans ses articles sur Palmerston, ainsi que les débats dont nous trouvons dans ce cahier de longs extraits: Communes, 24 août 1823,28 et 29 août 1833,7 mars I 834 , I I juillet 1835,20 avril 1836,17 mars 1837,21 juin 1838 , Ier juin I 840,13 , I 5 et 20 juillet I 840,6 août I 840,26 janvier I 841 , 20 septembre I 842,6 août I 846; Chambre des Lords: 4 février I 834, 26 janvier 184 (entreprises russes: Sandjak, Baltique, Pologne, Turquie, etc.). Nous y trouvons également des correspondances de Palmerston, Nesselrode, Thiers; des extraits du Traité de Londres du is juillet I 840; du Traité relatif à la succession de la Couronne de Danemark (Londres, 8 mai 18 ;2).

Plus de la moitié du cahier est rempli d'extraits des trois ouvrages suivants:

Geo. Fr. v. Martens, Grundriss einer diplomatischen Geschicbte der europäischen Staatshändel... x 807. Les passages recopiés concernent plus particulièrement la période de 1300 à I660. Ils trouvent leur continuation dans un autre cahier non daté, que nous examinons plus loin. 
De l'ouvrage de David Urquhart, Progress of Russia in the West, North and South, $2 \mathrm{~d}$ ed. London $x_{5} 3$, sont extraites une quinzaine de pages. Une phrase notée en passant nous indique une préoccupation familière de Marx: «[Russia] has to work out a war between England and France and a European revolution." 1

Une dizaine de pages, enfin, sont consacrées à:

G. K. Francis, Opinions and Policy of Mr. Palmerston (London 1852 ). ${ }^{2}$

L'intérêt que Marx prit à ces lectures et études historico-diplomatiques ressort de ses lettres à Engels de 1853 -1 8 54. Il s'estimait heureux d'avoir fait porter ses investigations sur la carrière politique de Palmerston (ce qui lui avait permis d'arriver à la même conclusion que D. Urquhart, à savoir que le diplomate anglais «est vendu depuis plusieurs décennies à la Russie»), d'avoir pu examiner de près l'histoire de vingt années de diplomatie. ${ }^{3}$

$$
\text { ** } *
$$

Non daté, le cahier suivant se rattache par son contenu au précédent et appartient, par conséquent, à la période de fin 1853 aux premiers mois de I $854 .^{4}$

Ses soixante-dix pages sont consacrées principalement à deux sujets historiques: $1^{0}$ les événements de 1839 au Moyen Orient qui conduisirent au rapprochement russo-anglais et à la Quadruple Alliance pour la protection de la Turquie, à l'exclusion de la France; $2^{0} l^{\text {'histoire de }}$ l'Europe, particulièrement du Nord et de l'Est, de la paix de Roeskild (I658) au traité russo-turc de Koutchouk Kainardji (i 774).

Pour le premier sujet, nous relevons aux folios I à 37 une abondante moisson de documents diplomatiques tirés d'une collection off icielle ${ }^{5}$ intitule Correspondence relative to the affairs of the Levant. Presented to both Houses of Parliament 1841 .

Les signataires de ces lettres et les noms qui reviennent dans ces documents sont ceux des diplomates et des militaires des grandes

\footnotetext{
1 Marx en cite un passage dans son sixième article sur Palmerston, People's Paper, ro. I 2. 1853. GS I, p. 27I. Le rôle joué par Chateaubriand au Congrès de Vérone est précisément celui que $D$. Urquhart (o.c., p. 4 o sq.) suggère à Marx.

2 Voir les huit articles de Marx sur Palmerston publiés simultanément dans People's Paper d'octobre 1853 à janvier 1854. GS I, p. 224-288. En français, Karl Marx, Oeuvres politiques, t. I, trad. J. Molitor, éd. Costes, Paris I929. Dans son second article, Marx cite quelques passages de la brochure de J. Mac Neil, Progress and Present Position of Russia in the East (1836). Aucun des cahiers vus par nous en porte une trace.

3 „Nous avons trop négligé ce point”, écrit-il le 2.11.1853 à Engels, „et il faut savoir à qui on a affaire." Déclarations semblables dans ses lettres à Lassalle (6.4. I 854: Marx résume en quelques lignes la carrière de Palmerston, - au service de la Russie; et 3.5.1854).

4 Classé actuellement sous B 76 , avec la mention peu vraisemblable „, 1856 ”.

${ }^{5}$ A moins qu'il ne s'agisse du Portfolio d'Urquhart que nous rencontrons plus loin aux folios $58-62$.
} 
puissances européennes mêlées au conflit turco-égyptien de 1839: Molé, Granville, Palmerston, Nesselrode, Cochelet, Soult, StirnerAlexandre, Metternich, Pozzo di Borgo, Campbell, Russell, etc.

Quant au second sujet, il est représenté exclusivement par des passages tirés de l'oeuvre de Martens, rencontrée au cahier précédent. ${ }^{1}$ Les cahiers se terminent par une page d'extraits relevés dans l'ouvrage de l'abbé Pierre Joubert, Histoire des révolutions de Pologne depuis la mort d'Auguste III jusqu'à l'année I775... Varsovie, 1775.

Le cahier qui semble faire suite au précédent (à supposer qu'aucun autre cahier n'a été perdu) porte la date de Londres 1854, "mai-juinjuillet». ${ }^{2}$ C'est le premier d'une série de quatre cahiers consacrés surtout à l'histoire des révolutions espagnoles.

En tête de ce cahier figure l'ouvrage de Henri Healdy Parish («the late secretary of Legation to Greece"), The Diplomatic History of the Monarchy of Greece, from the year $1830 .$. London 1838 . Marx en a recopié, sur neuf pages, des documents diplomatiques concernant principalement les rapports de la Grèce avec la Russie et le rôle de l'Angleterre. ${ }^{3}$ En passant, il a noté deux titres: F. Thiersch, $L$ 'état actuel de la Grèce... etc., 2 vol., Leipzig $1833 ;{ }^{4}$ Georg Ludwig Maurer, Das griecbische Volk... Heidelberg 1835 .

\section{LES RÉVOLUTIONS D'ESPAGNE}

Ce sont les mouvements insurrectionnels en Espagne, dont les premières manifestations étaient apparues dès février $18 \$ 4$, qui attirèrent alors l'attention de Marx. ${ }^{5}$ Après l'insurrection militaire près de

${ }^{1}$ Folio 37. Interrompus au folio 57 , les extraits de Martens réapparaissent aux folios 64-68.

${ }^{2}$ Classé actuellement sous $B 68$. Il est probable que des cahiers antérieurs à cette date manquent. En effet, dans sa correspondance de mars à mai 1854, Marx mentionne des lectures dont les cahiers postérieurs ne font pas état, par ex.: Jos. v. Hammer-Purgstall, Geschichte des Osmanischen Reiches, 2e éd. Pesth, I834-I836; cf. M. à E., 29.3.1854. W. Wilks, Parlmerston in the three epochs, London, 1854; cf. M. à E., 4.4.1854 (Marx considéra ce livre comme un plagiat de ses articles de la NYT).

${ }^{3}$ Dès mars I 854, Marx avait commenté en plusieurs articles les mouvements insurrectionnels en Grèce. Il en attribuait l'origine aux intrigues russes. Voir NYT, I3, I 8 et 29 mars 1854. Les événements font également l'objet d'articles parus les 2,6 , Is et I6 mai I 854 . Dans sa lettre à Lassalle du 1.6.18 54 , Marx invoqua le témoignage de Parish pour affirmer qu' „à partir de 1830 , Palmerston a tout fait pour transformer la Grèce en une province russe." Voir également sa lettre à E., 3 mars I 854, où il dit qu'il est en train de „bûcher" l'histoire de l'empire néohellénique.

4 Dans sa lettre du 9.4.1857, Marx recommande à Engels, qui recherche des „Anti-Palmerstoniana", entre autres les livres de Parish et de Thiersch.

${ }^{5}$ Le pronunciamento de Don Juan de Hore eut lieu à Saragosse le 20 février 1854 . Cf. Riazanov, GS II, p. 546 sq. Voir son article dans la NYT, x8.3.1854, GS, I, p. 355 sq. Marx rappelle l'intervention française en Espagne en 1823 et l'intervention franco-anglaise de 1834 et les rôles respectifs de Chateaubriand et de Palmerston. 
Madrid, conduite par le général Espartero, Marx envoya de nombreuses correspondances à la NYT dans lesquelles les événements espagnols sont longuement commentés. ${ }^{1}$ Il apprit l'espagnol «à ses moments perdus», en lisant Calderon et (en espagnol!) Atala et René de Chateaubriand. ${ }^{2}$

$$
* *
$$

Le cahier daté de mai-juillet $\mathrm{x} 854$ contient à partir du folio ro des extraits et des notes de lecture sur une quarantaine de pages, relatifs à l'Espagne. Voici des titres:

Florez (D. José L.). Espartero. Historia de su vida militar y politica y de los grandes sucesos contemporaneos etc, $2 \mathrm{e}$ éd., $4 \mathrm{vol}$ ( (1844/45) ${ }^{3}$

Notes of an attacbe in Spain. In 1850 . London 1851.4

Una (plumada) sobra le Indole y Tendencia de Revolucion en España. Madrid I 846.5

Révolution d'Espagne. Examen critique. I820-1836. Paris 1836. Très nombreux passages recopiés aux folios io à 12 et 38 à 48 .

Principe y Vidaud (Miguel Agustin), Espartero, su pasado, su presente, su provenir, etc. ze éd. Madrid $1848 .^{6}$

The Crisis of Spain. 2e éd. London 1823 . Plus de quatre pages d'extraits sur l'organisation politique et administrative de l'Espagne, etc.

Revolutions of Spain in I 845. By an English Resident. 2 vol. London 1845. Nombreux extraits de presque tous les chapitres des deux volumes. Cet ouvrage très riche semble avoir été la source principale de Marx pour ses correspondances sur Espartero et sur la structure politique et économique de l'Espagne.

En fin de copie, les titres suivants ont été notés:

Toreno (Conde de): Historia del levantiamiento, guerra y revolución de España, 5 vol. Madrid I855. (Voir le cahier suivant).

A. Custine (Marquis de), L'Espagne sous Ferdinand VII, Paris, $1838 .^{7}$ Mariana (Juan de), Historia general de España... completada per Florida Blanco ... y ... E. Cbao. Madrid 1849-18 $50 .{ }^{8}$

Autre titre noté au passage:

${ }^{1}$ Voir BIBL. 325, 328, 332, 333, 337, 338. Rédition dans REV. SPAIN.

${ }^{2} \mathrm{M}$. à E., 3.5.I854. Dans la même lettre, il mentionne l'Archivio triennale delle cose d'Italia dall'avenimento di Pio IX all abbandono di Venezia etc. „C'est ce qu'il y a de mieux que j’aie lu du parti révolutionnaire italien." Le 3.6.18 54 , Marx en a terminé la lecture.

3 Mentionné par Marx dans son article sur Espartero, NYT, 12.8.1854 (éditorial). Cf. REV.SPAIN, p. I03 sq.

4 Note de Marx: „Reiner Kohl..."

5 Note de Marx: „Dominikaner Wisch..."

- Mentionné par Marx dans Espartero, NYT, i 2.8.1854, cf. REV. SPAIN, p. 108.

"Note de Marx: „belletristisch ekelhaft!"

${ }^{8}$ Note de Marx: „In Progress”. L'ouvrage est en cinq volumes, Madrid, r847-18 1 I. 
Martignac (Jean Baptiste vicomte de), Essai bistorique sur la révolution d'Espagne et sur l'intervention de 1823 , Paris, I $832 .{ }^{1}$

L'ouvrage suivant semble avoir été lu avec la plus grande attention: M. de Marliani, Historia politica de la España moderna. ${ }^{2}$

Une page d'extraits du chapitre $\mathbf{I}^{3}$

Il est probable que l'étude de l'histoire espagnole et spécialement des Cortes ait conduit Marx à s'intéresser plus particulièrement à l'histoire des municipalités médiévales. ${ }^{4} \mathrm{Ce}$ qui pourrait expliquer la présence, dans ce cahier plutôt "espagnol», de près de quinze pages remplies d'extraits, recopiés en français ou résumés en allemand, du livre d'Augustin Thierry, Essai sur l'bistoire de la formation et des progrès du tiers état etc. Paris, $1853 .^{5}$

Marx a retenu tout ce qui touche les Etats Généraux et les rapports du Tiers-Etat avec la royauté sous Louis XIV. Le Tableau de l'ancienne France Municipale, les dispositions de la charte communale d'Amiens tout comme le statut des établissements communaux dans les diverses régions, avant et après le XIIe siècle sont notés avec beaucoup de soin, mais apparemment sans aucun commentaire.

$$
* *
$$

Tournons-nous maintenant vers les trois cahiers «espagnols» mentionnés ci-avant comme étant de 1854 .

Un cahier daté «London, août I 854 ", d'une quarantaine de folios, fait partie du même groupe. ${ }^{6}$ Il s'ouvre sur une suite d'extraits remplissant près de six pages: ce sont les articles de la Constitution espagnole de I812, en une version anglaise:

The Political Constitutes of the Spanish Monarchy. Proclaimed in Cadiz, 19 March, 1812 . London $1812 .^{7}$

' Marx mentionne (Fol. 50) cet ouvrage sous son titre espagnol La Espana y sus Revoluciones, I833. Dans son article de la NYT, 2.I2.1854, il cite cet ouvrage sous le titre L'Espagne et ses Révolutions (1 832) et reproduit le passage extrait.CF.REV.SPAIN,p. 83. ${ }^{2}$ Marx note que le livre parut en français à Paris, $184_{2}$. Il en cite un passage (trad. de l'espagnol) dans un article de la NYT, r.9.1854. Cf. REV. SPAIN, p. I I 5. Concerne la légion formée par Napoléon avec des prisonniers espagnols qui passèrent, après la défaite, dans la camp russe.

${ }^{3}$ Les extraits seront repris dans un cahier non daté, classé sous B 69.

- Voir par exemple, ses huit articles sur l'Espagne révolutionnaire parus dans la NYT du 9 septembre au 2 décembre 1854 . REV. SPAIN, p. I 92 sq. particulièrement le premier et le sixième.

${ }^{5}$ Sur cette lecture, voir la lettre de M. à E. du 27.7.1854. Les remarques analytiques et critiques sur l'ouvrage de Thierry, "père de la lutte des classes" dans l'historiographie française sont d'un grand intérêt pour la connaissance des sources dont Marx a tiré sa conception du socialisme communaliste.

${ }^{6}$ Classé sous B 70 , il est sans doute antérieur à B 69 où nous trouvons la suite de certains extraits commencés dans celui-li.

7 Marx s'est servi de ces extraits pariculièrement dans son article paru dans la NYT, 24.II.I 854. REV. SPAIN, p. 56-68. 
Suit un titre noté sans extraits :

Ch. Romey Histoire d'Espagne .. 8 vol. Paris 1839-1848.

Puis treizepages remplies d'extraits recueillis dans les trois volumes de:

A. Southey, History of the peninsular War. 3 vol. London, 1823-1832.1 Du même auteur, Marx a copié d'autres passages aux folios 23-24. Cet ouvrage est suivi d'un titre français:

A. Du Casse, Mémoires et correspondance politique et militaire du Roi Joseph etc., ro vol., Paris 1853-1854. Les extraits débutent au t. IV, p. 246 (pour l'année I 807) et couvrent deux pages et demie.

Suivent quelques articles recopiés de la Constitution décrétée par l'Assemblée Constituante, 3 sept. 1791. Déclaration des droits de l'homme et du citoyen. ${ }^{2}$

Aux folios 25-26, nous trouvons:

J. Bigland, Histoire d'Espagne depuis la plus ancienne époque jusqu'à la fin de l'année $1809 \ldots$, traduite de l'anglais, continuée etc.... par le comte Mathieu Dumas etc., 3 vol. Paris, 1823 .

Ces extraits commencent au vol. III, c'est à dire après $\mathrm{I} 809$, et s'arrêtent à la date du I9 avril 18 Io.

Au folio 26, titres de trois ouvrages de Don José Clemente Carnicero, datés de 1814 et 1816 :

Historia razonada de los principales sucesos de la gloriosa revolución de España... 4 vol. Madrid I 814.

La Inquisición justamente restablecida, Madrid 1816.

Napoleón, verdadero Don Quijote de Europa. ${ }^{3}$

Suit un auteur anglais dont l'ouvrage est abondamment exploité (près de neuf pages d'extraits du rer volume):

W. Walton, The Revolutions of Spain. 1808-1836, 2 vol. London 1837.

$\mathrm{Au}$ folio 35 , nous rencontrons de nouveau le nom de Marliani, suivi sur les dix pages qui terminent le cahier de:

Conde de Toreno, Historia del levantamiento, guerra y revolución de España. Madrid I 835 -1 $837 .^{4}$

Les extraits sont pris surtout des volumes II et III, relatant les événements de i 808-1810.

$$
* *
$$

Nous avons maintenant un cahier non daté, d'une quarantaine de folios dont les deux premiers manquent. ${ }^{5}$

${ }^{1}$ Marx en cite un passage dans l'article de la NYT, 20.10.1854. REV SPAIN, p. 31.

${ }^{2}$ Marx compare la Constitution espagnole de 1852 et la Constitution française de 1791 dans son article paru dans la NYT du 24.11.1854.

${ }^{3}$ Marx nomme cet auteur et ses trois ouvrages (avec leurs titres en anglais) dans son article de la NYT, 25.9. I 854 . Il ne semble pas en avoir dépouillé le contenu.

4 Il s'agit d'une "suite", comme le note Marx; en effet, nous rencontrons ce titre dans le cahier précédent, classé sous B 68.

5 Il est classé actuellement sous B 69 . 
Aux folios 3 et 4 , nous lisons la suite et fin des extraits d'un ouvrage anglais concernant des événements de 1820 à I 822 : la rencontre à Troppau des empereurs de Russie et d'Autriche avec des représentants de l'Ângleterre et de la Prusse, le Congrès de Laïbach et le Congrès de Vérone.

A partir du fol. 4 et jusqu'à la fin du cahier, nous avons une quinzaine d'ouvrages, en anglais, en espagnol et en français, se rapportant à l'histoire de l'Espagne moderne. ${ }^{1}$ En voici les titres:

D. Evaristo San Miguel, Memoria Succinta sobre lo Acaecido en la Columna Mavil de las Tropas Nacionales al Mondo del Comendante General de la Primera Division Don Rafael del Riego... Madrid 1820.

Ce mémoire fut utilisé par Marx pour son huitième article de la série qu'il envoya à la NYT pendant les dernières mois de $1854 .^{2}$ C'est le récit de l'extraordinaire expédition de Riego qui, avec sa colonne de 1.500 hommes formée contre la volonté de ses chefs, et ayant une force dix fois plus grande, traversa du 27 janvier au I I mars I 820 l'Andalousie et proclama la Constitution dans toutes les villes importantes de cette province. Dans son cahier, Marx note ironiquement (en allemand): «Toute la campagne décrite ici se réduit à une chasse à la nourriture», puis (en anglais): «Il n'existe peut-être pas de campagne plus curieuse que cette fameuse expédition patriotique", - dont San Miguel se fit l'Hérodote. Marx résume son jugement dans cette remarque: «L'Espagne avec tous ses défauts."

Letters from Spain by Don Leucadio Doblado.... London I 822. Note de Marx: «Pseudonyme de Blanco White».

Les extraits, souvent résumés en anglais et en allemand, concernent l'influence de la religion en Espagne.

Clarke (Christopher), An Examination of the Internal State of Spain at the Period of the Unions of the Crowns of Castil and Aragon, London I 8 I 8. Marx a retenu particulièrement les renseignements fournis par l'auteur (qui aurait "copié» G. M. de Jovellanos ${ }^{3}$ ) sur les conditions de la propriété rurale et l'agriculture, les communications, le commerce,

\footnotetext{
1Ma principale étude est maintenant l'Espagne", écrivait M. à E. le 2.9.1854. "J'ai bûché jusqu'à présent des sources espagnoles, l'époque de I808-1814 et I820-1823. J'en arrive maintenant à la période $1834-1843$. Cette histoire est assez embrouillée. En tout cas, $j$ 'ai bien fait de commencer à temps la lecture du Don Quichotte. Le tout fera environ six articles pour la Tribune si je réussis à condenser beaucoup. N'importe comment, quel progrès que d'être payé pour ses études!"

Marx donna effectivement neuf articles à la NYT qui n'en publia que huit, du 9 septembre au 2 décembre 1854. Voir BIBL. 337. Réédités dans REV. SPAIN, p. 19-84.

2 Il y parut le 2.I 2.1854. REV. SPAIN, p. 75-84.

${ }^{3}$ Sur Jovellanos, membre de la Central junta, voir l'article de Marx dans la NYT, 20.10. I 854, REV. SPAIN, p. 32 sq.
} 
les impôts, l'Eglise, les colonies, etc. L'histoire de l'Espagne y occupe également une place importante.

John Bramsen, Remark on the North of Spain, London 1823.

The Last Days of Spain... by an Eye-witness. London 1823.

C'est une apologie de la monarchie et de l'Eglise espagnoles. Marx en retient les figures et les partis politiques ayant joué un rôle dans les événements de 1819 et 1820.

La España Bajo el Poder Arbitrario de la Congregacion Apostolica... de I 820 à I 832 . 2e édition, Paris 1833 .

Les extraits et passages traduits en allemand, couvrent six pages. Marx a dû retirer de cet ouvrage une connaissance approfondie de la situation politique et économique de l'Espagne pendant les années considérées.

Who is the Liberator of Spain? An Essay towards deciding the question, published at Sevilla, July, I820. Transl. from the original by Henry Wood. London 1846 . L'équipée de Riego vue par un patriote espagnol.

The Holy Alliance versus Spain etc. By a Constitutionalist, London I 823. Chateaubriand, Congrès de Vérone, Guerre d'Espagne, etc. Paris 1838. Marx semble s'être livré, en lisant cet ouvrage, à un travail de détective: il s'agissait de saisir le rôle exact assumé par Chateaubriand au Congrès de Vérone. Dans sa correspondance datée du I s.8.1854, où il examine la politique russe dans les affaires d'Espagne, Marx ne semble pas encore avoir résolu cette question; il se borne en effet à la résumer en ces quelques mots: «Or, nous savons par Chateaubriand, ambassadeur de France au Congrès de Vérone, que la Russie incita l'Espagne à entreprendre l'expédition en Amérique du Sud et qu'elle força la France à se livrer à une expédition en Espagne.» ${ }^{1}$ En revanche, cette lecture fut remarquablement commentée par Marx dans une lettre à Engels. ${ }^{2}$

Observations sur l'état actuel de l'Espagne (1825).

De l'Espagne au mois de Mars I 836 .

Trois pages d'extraits de la «Revue d'Espagne», articles anonymes(?), sans autres indications.

\section{Marliani, Historia politica de la España moderna, etc. 1849 .}

Deux pages d'extraits qui sont la suite des notes, en espagnol et en allemand, commencées dans le cahier, déjà examiné, daté de maijuillet. Nous en retrouvons une troisième dans un cahier suivant, daté de novembre 1854 .

${ }^{1}$ NYT, 1.9.1854, REV. SPAIN, p. II4 sq.

${ }^{2}$ Elle date du 26.ro.r854. Nous y tenons l'explication du sentiment d'aversion que Marx a exprimé en maintes circonstances non seulement à l'égard de Chateaubriand, homme politique, mais aussi envers l'homme de lettres. Marx recopie, à l'intention de son ami, plusieurs extraits notés dans son cahier, où Chateaubriand se révèle un instrument docile de la diplomatie russe, ne reculant pas devant le mensonge et le double jeu. 
Walton, The Revolution of Spain, etc. Reprise des extraits commencés au cahier daté de août 1854 , vu plus haut.

V.-A. Du Hamel, Historia constitucional de la Monarquia Española... Note de Marx «Traduit en espagnol par Baltasar Andruaga y Espinosa, 2e éd., 2 vol. Madrid I 848 .» 1

Au folio 38 , nous lisons ce titre:

Carta del etc. D. José Calatrava etc. Réponse[?] de Don Alvaro Florez Estrada. London I 825 . Une page et demie d'extraits où figure souvent le nom de Riego.

Au folio 40 , suite des extraits de Marliani, puis, sur les six dernières pages de ce cahier:

Michael J. Quin, $A$ Visit to Spain, etc., London 1823.

Marx en a retenu particulièrement les renseignements sur l'état économique et financier de l'Espagne de $182 \mathrm{I}-1822$.

De la même époque, mais sans précision chronologique, date un cahier d'une vingtaine de pages, que Marx semble avoir "emprunté" à sa fillette Laura, si nous en jugeons d'après la lettre-brouillon qui y figure aux premières trois pages: l'enfant remercie en allemand sa grand-mère pour les jouets qu'elle avait reçus. ${ }^{2}$

Ce n'est qu'au folio 5 (folioté $\mathrm{I}$ ) que nous rencontrons les premières notes de Marx; il s'agit sans doute du brouillon d'un article destiné à la NYT, commentant une junte centrale réunie en septembre i 808 à Aranjuez. Les événements relatés se rapportent à l'invasion des troupes napoléoniennes, la capitulation de Bailen et l'évacuation de Madrid par Joseph Bonaparte. ${ }^{3}$

Après ces notes sur une page et demie, Marx recommence le foliotage et inscrit sur six pages des extraits de Toreno (vol. III et IV), qui font suite à ceux que nous avons rencontrés au cahier précédent. Ce sont les événements d'Espagne, en I 8 Io- 8 I I , qui y retiennent son attention. Nous retrouvons la suite de ces notes aux folios Io à 13 , puis au folio 17 , avec les années $18 \times 2$ à 1814 , Marx a consulté plusieurs ouvrages à la fois, car entre ces groupes d'extraits de Toreno, nous trouvons aux folios 7,8 et 16 des passages recopiés des Mémoires bistoriques sur la Révolution d'Espagne (Paris 1 816 ) de l'Abbé de Pradt, que Marx a abondamment utilisés pour ses articles.

Il s'agit, dans ces extraits, de la campagne française de 1808 et de la résistance farouche du peuple espagnol, harcelant sans cesse l'enva-

${ }^{1}$ Marx reprendra cet auteur dans un cahier daté de novembre I854. Titre de l'édition originale: Histoite constitutionnelle de la monarchie espagnole, depuis l'invasion des hommes du Nord jusqu'à la mort de Ferdinand VII. Paris 1845 .

Classé sous B 7 I.

${ }^{3}$ Manifestement, ces notes sont destinées à la série d'articles publiés par Marx dans la NYT de septembre et décembre I 854 . Voir REV.SPAIN, p. I9 sq. et particulièrement l'article du $25 \cdot 9.1854$, ibid., p. 28 sq. 
hisseur désespéré; les guerrillas facilitées par la configuration du terrain; les difficultés des communications; l'isolement des habitations, le manque d'abris sûrs et de subsistances. ${ }^{1}$ Voici un passage noté et résumé par Marx: «Point d'armistice, de quartiers d'hiver. En Espagne, espèce de Vendée, les attaques partielles, les surprises, les enlèvements des convois, des postes isolés, des courriers et des escortes étaient journaliers. On n'a plus cessé de combattre, pas un jour ne restant écoulé sans que le sang coulât, et cela sur toute la surface de l'Espagne, depuis Cadiz jusqu'à Pampelune, depuis Grenade jusqu'à Salamanca, et l'usage de faire des prisonniers avait disparu...».

Dans un autre extrait, Marx notera cette phrase de l'Abbé de Pradt: «L'Espagnol est le Parthe de l'Européen.»

Sur les guerillas, Marx a consulté en outre un ouvrage au titre curieux: The two Alinas and the Spanish Guerillas. Extracted and translated from a work «On Spain», written by Capt. H. v. Brandt, a Prussian officer who served in one of the Polish regiments attached to the French Army during the Peninsular War. By a British Officer. London 1825. Extraits sur cinq pages et demie, rappelant l'appui apporté par les guérilleros à Wellington; la tactique de rassemblement et de dispersion des bandes, leur organisation, leurs chefs, l'obstacle fatal qu'elles ont été pour les Français. ${ }^{2}$

$\mathrm{Au}$ folio $\mathrm{I} 6$, le nom de l'Abbé de Pradt réapparaît avec un autre ouvrage: De la Révolution actuelle de l'Espagne et de ses suites. Paris 1820. Les passages notés par Marx concernent l'armée espagnole et certains des chefs révolutionnaires d'origine populaire. ${ }^{3}$

Les derniers folios du présent cahier ( 2 I à 25 ) sont entièrement réservés à un document consacré aux problèmes de la propriété foncière et de l'agriculture espagnoles:

Jovellanos, Obras. Barcelone I839-1840, publié par D. Venceslao de Linares y Pacheco. 8 volumes. ${ }^{4}$ Cinq pages d'extraits du $7 \mathrm{e}$ volume.

$$
* *
$$

Nous passons à un cahier daté de novembre 1854 et consacré comme ce qui précède aux questions espagnoles. ${ }^{5}$

Plus de huit pages représentant l'ouvrage de Victor Du Hamel, Historia Constitucional de la Monarquia Española, etc. Madrid 1848, $2 \mathrm{e}$ éd. ${ }^{6}$

\footnotetext{
1 Voir le texte et les notes dans ce cahier et repris dans les articles de la NYT de septembre et d'octobre 1854, REV.SPAIN, 19 sq., 50 sq.

2 Voir les articles dans la NYT, 27 et 30 octobre 1854

3 Sur le rôle joué par Enrique O’Donnel, Comte de La Bisbal, mentionné dans ce cahier, voir l'article dans la NYT, 2 décembre 1854 . REV. SPAIN, p. 75 sq.

4 Cité par Marx, NYT 25.9.1854. REV.SPAIN, p. 32.

5 Classé sous B 72.

6 Nous l'avons rencontré dans le cahier non daté, classé sous B 69 .
} 
Vient ensuite les: Fastos Españoles o Efemérides de la Guerra Civil desde Octobre de 1807 . Madrid 1839, ${ }^{1}$ et nous retrouvons l'abbé de Pradt avec les Garanties à demander à l'Espagne, Paris 1827 . Chartede Ferdinand VII «...que jamais il ne se départira du pouvoir absolu dont le ciel l'a rendu dépositaire...»; et cette citation des Mémoires du Marquis de Louville attaché à la personne de Philippe V, que les autres personnes de la cour avaient «un seul ange gardien, mais que les rois en avaient deux, dont l'un pour présider au gouvernement de leur Etat."; citation des Mémoires d'Ouvrard, sur une junte convoquée "pour prononcer: si un roi est tenu de payer les dettes de son prédécesseur»; et que l' «armée de la Foi est devenue proverbiale pour tout ce qui est lâche, sale et vil...»

D. Evaristo San Miguel. De la guerra Civil de España. Madrid r 836: sur le statut royal, Ferdinand VII et les Cortes.

An Historical Inquiry into the Unchangeable Character of a War in Spain. London i 837. «... The remark of Polybius (L.IX) that a war in Spain was very different from those in Greece or Asia, where one great battle decided the campaign, whereas here it was one endless, uninterrupted series of contests; [...] as Strabo observed, they unlike the Gauls split themselves into small bands, and kept up a desultory or robber or, in one word, a guerilla warfare (Strabo IV) [...]». Passages copiés sur la cruauté des Espagnols, sur leur apparence de dignité, avec un véritable registre de leurs défauts; vénalité et concussion chez les généraux espagnols, etc.

L'Espagne et la Crise Présente. Examen raisonné de la cause et des bommes qui peuvent sauver cette nation, ouvrage écrit en espagnol [...] traduit par G. D. Montpellier (jésuite). Sur l'affaiblissement du pouvoir, le gouvernement christino-libéral ou juste-milieu, dont l'auteur réactionnaire condamne la tolérance en matière de religion et de morale, etc.

Le reste du cahier offrira, avec de nouveaux extraits de Marliani, déjà cité, de nouvelles pages de Quin, Visit to Spain, etc. London I 823 : Chronologie détaillée du printemps de 1823 ; Chateaubriand; O'Donnell; levée de milices; accueil favorable aux institutions libérales chez les étudiants et les commerçants, etc. Des chiffres sur les importations et les exportations espagnoles, sur les propriétaires écclésiastiques et laïques, etc. Annotation sur: Marques de Miraflores, Memorias para escribir la bistoria contemporanea de los siete primeros años del Reino de Isabel II, 2 vol., Madrid 1843 et 1847. Du même auteur: Apuntes bistórico-criticos para escribir la revolución de España, 1820-1 823, London I834. L'annotation se poursuit en allemand sur la Quadruple Alliance dirigée par Palmerston contre la France. Enfin, une référence à l'Edinburgh Review, vol. XXXVIII, et XXXIX (où se trouve une 1 Note de Marx: „Tag für Tag die Zeitgeschichte”. 
étude de Quin sur les événements d'Espagne); et des articles recopiés du Traité de Veliki Louki (20 juillet I 8 I 2) entre la Russie et les Cortes d'Espagne. ${ }^{1}$

Les derniers folios contiennent des chiffres tirés de l'Economist ( 6 janvier 1858) sur les importations et exportations en I 852 , 1853 et 1854; sur les fluctuations du marché, les augmentations de prix dans le transport maritime, etc. Ces indications sont recueillies en vue d'un article dont le début se trouve à l'état de brouillon au dernier folio (la crise commerciale qui menace l'Angleterre).

$$
* *
$$

Il conviendrait de mentionner ici un cahier non daté qui semble avoir été utilisé à deux époques différentes, séparées peut-être par un intervalle de quelques années. ${ }^{2}$

En effet, nous y trouvons tout d'abord aux folios numérotés 3 à 5 (les folios $\mathrm{I}$ et 2 étant vides) le brouillon d'un article inédit sur les événements révolutionnaires de 18 20-1 823 en Espagne; puis, sur une vingtaine de folios, numérotés de I à 23 , un ensemble de notes en allemand, parsemées de citations en anglais et groupées sous le titre "Geldwesen, Creditsystem, Krisen-Citate." ${ }^{3}$ Après deux pages blanches, nous avons, sur deux folios, des notes statistiques se rapportant à l'histoire commerciale de Venise et de Trieste, suivies du brouillon fortement raturé d'un article intitulé "The War against Persia».4

Le texte sur l'Espagne est à rapprocher des articles écrits par Marx sur les années révolutionnaires de $1820-1823$, qu'il compare ici aux événements de $1833-1843$, tout en formulant des jugements généraux. Voici un exemple:

"The positive results of the Revolution of $1820-3$ were not limited to the great fermentation which expended the minds and received the characters of some large classes of the nation. The second restauration, in which the antiquated elements of society assumed[?] such shares as to become insupportable to, and incompatible with the national existence of Spain, was itself a produce of the Revolution. Its principal work was to whet the antagonism to such a point as to make all

${ }^{2}$ Voir NYT, 2.1 2.1854. REV.SPAIN, p. 8 I.

2 Classé actuellement sous $\mathrm{B} 79$.

${ }^{3}$ Dans GR, une note des éditeurs fait mention de ce texte en tant que manuscrit rédigé vers la fin de 1854 . Cf. GR, p. I044. Il y aurait lieu de penser qu'il date de 1856 à 1857 , Marx ayant seulement alors repris le travail pour son oeuvre économique.

4 Ce sujet fut traité par Marx et par Engels dans plusieurs articles publiés dans la NYT en 1857. Voir M. à E., 30-10.1856 et BIBL. 460, 474, 476. Le brouillon débute par ces mots: "The Persian War is the rehearsel of a diplomatico-military drama for the first time performed by Lord Palmerston more than twenty years ago." C'est le thème familier de Marx: Palmerston, instrument de la politique russe. 
compromises impossible and a war to the knife inevitable. According to Lord Liverpool himself, there never was an extensive political change attended with less violence or bloodshed than the Spanish Revolution during 1820-3, when we be told therefore, the civil war of $1833-43$, exterminating the antiquated elements of Spain. Society, - with fire and sword and disgracing itself by acts of cannibalism, we must not attribute the savage inexorableness of that epoch to the peculiar character of the race, but to the same force of circumstances that imposed upon France the reign of terrorism. While the French centralised and, therefore, abbreviated the reign of terrorism, the Spaniards, true to the traditions, decentralised and, consequently, procrastinated it.»

Nous reviendrons à ce cahier lorsque nous aborderons l'examen des matériaux de la période de 18 57-1861, date de la reprise par Marx de son oeuvre économique.

\section{HISTOIRE DIPLOMATIQUE}

Pour 1855 , aucun cahier ne semble être conservé à Amsterdam. ${ }^{1}$ Quelle fut l'activité de Marx pendant cette année? Principalement journalistique. Peu d'articles pour la NYT. ${ }^{2}$ En revanche, un journal allemand, la Neue Oder-Zeitung, sollicite sa collaboration. Marx, aidé par Engels, donnera, de janvier à décembre 1855 , plus de 150 articles politiques à ce quotidien libéral. Ce sont souvent des traductions des articles de la NYT. ${ }^{3}$ Il lit comme d'habitude, mais semble être las du travail de copie. Il reprend la lecture de ses cahiers économiques et prépare des matériaux pour les articles militaires qu'Engels écrit pour Putnam's Magazine. ${ }^{4}$

Pour 1856, nous avons plusieurs cahiers, pour la plupart datés. Presque tous contiennent des matériaux étudiés par Marx en vue d'une oeuvre plus importante qui devait avoir pour thème les relations

${ }^{1}$ La correspondance M.-E. de 1854 et 1855 révèle pourtant que Marx a fait diverses lectures: Ripley, Mexican War. Antonio de Soli, Conquista de Mexico. Sur ces deux livres, voir ses remarques à Engels, 30.1 I et 2 et I S.I 2.1854.

' C'est une année difficile, voire tragique pour Marx et pour sa femme: maladie et mort de leur garçon; embarras financiers; difficultés avec la NYT; Engels se substitue à son ami et écrit pour Putnam's Magazine. Marx passe quelques temps en Ecosse. Voir M. à E., 12.4.1855.

' Guerre de Crimée, crise en Prusse, politique intérieure et extérieure de l'Angleterre, alliance franco-anglaise, situation intérieure du second Empirc, les réfugiés politiques en Angleterre, situation de la Russie, etc. Sur la collaboration de Marx à la NOZ, voir Stanistaw Schwann, Korespondencje Karola Marksa Do Wroclawskiej „Neue OderZeitung", Poznan Institut Zachodni, 1958.

"En lisant ses propres cahiers, Marx a contracté une maladie des yeux. Lettre à E., 13.2 . 1855. Allusion à d'autres lectures (sur Napoléon, l'histoire romaine, etc.) dans sa lettre du 8.3.1855. 
diplomatiques entre l'Angleterre et la Russie du XVIIIe siècle à son époque. Il n'en rédigea que l'Introduction qu'il publia sous le titre Revelations of the Diplomatic History of the Eighteenth Century dans The Free Press de D. Urquhart. ${ }^{1}$

Pour cette introduction, Marx a fait largement usage de ses cahiers remplis en 18,6 . Ils prennent de ce fait une importance particulière puisqu'ils permettent d'assister pour ainsi dire visuellement à la composition de ce curieux pamphlet antirusse.

$\mathrm{Au}$ demeurant la correspondance Marx-Engels nous renseigne assez complètement sur les sources utilisées par Marx.

Rappelons qu'à ses yeux, la défaite militaire de la Russie en Crimée semblait se terminer par une nouvelle victoire diplomatique: la fin de l'hégémonie russe en Europe n'était pas proche. Les matériaux étudiés lui révélèrent l'origine de cette hégémonie, acquise avec l'appui de l'Angleterre, et notamment des whigs.

Il informa aussitôt Engels des "découvertes historiques» qu'il avait faites au British Museum sur la guerre nordique et le rôle de l'Angleterre. Il résume ainsi son jugement: «La politique extérieure des Tories se distinguait de celle des Whigs simplement en ceci, que les premiers se vendaient à la France, les seconds à la Russie».2

Puis Marx énumère ses sources, et nous retrouvons exactement les mêmes titres, à peu près dans le même ordre, sans commentaire, dans un cahier dont Marx s'est borné à noter l'année de rédaction: $1856 .^{3}$

Sur la couverture intérieure, Marx a noté le sommaire (1o titres) de ce cahier qui comprend 26 folios.

Le premier folio donne un passage de douze lignes tiré du Mémoire présenté à sa Majesté britannique, par Wesselowsky, Ministre de Sa Majesté Czarienne, London, $1717^{4}$

Sur la même page, orientée verticalement, une table généalogique des maisons régnantes en Russie, depuis Ruric $(\nmid 878)$ à Nicolas Ier

1 Voir BIBL. 457. Sur cette Introduction, voir Herr Vogt.

Réédition des articles par Eleanor Aveling sous le titre: Karl Marx, Secret Diplomatic History of the Eighteenth Century, I 899.

V. Riazanov, Karl Marx über den Ursprung der Vorherrschaft Russlands in Europa. Ergänzungshefte zur Neuen Zeit, no s, mars I 909.

Trad. française: Karl Marx, La Russie et l'Europe. Première trad. complète, précédée d'une introduction par B. P. Hepner, Paris, 1954.

${ }^{2}$ M. ̀े E., le I2.2.1856.

${ }^{3}$ Classé actuellement sous B 74 .

4 Dans sa lettre à E., M. fait suivre ce titre du commentaire suivant: „Verteidigungsschrift der Russen wegen ihrer Mogeleien mit dem Prätendenten, besonders merkwürdig, weil schon ganz der Stil (obgleich noch nicht ganz die Glätte) der Pozzo di Borgo und Konsorten und so Beweis, daß kein qualitativer Fortschritt in der russischen Diplomatie seit Pierre Ier." Ce document n'est pas mentionné dans Révélations. 
(† 1855), tirée de: A Chapter on Russia and on the Diplomatic Service. London I 850 . By the Hon. Perceval. ${ }^{1}$

Puis, sur les folios I à 5 , des extraits d'un ouvrage au titre curieux que voici: Truth is truth, as it is timed, or Our Ministry's present Measures against the Moscovite vindicated by Plain and Obvious reasons, tending to prove that it is no less the interest of Our British Trade, than that of Our state that the Cqar be not suffered to retain a Fleet - if needs must that be should bave a Sea Port in the Baltic, etc., London I7I9.

Les passages extraits et annotés (en allemand) sont reproduits dans les Révélations. ${ }^{2}$ Ces notes sont suivies, sur les folios 5 à Io, d'extraits du document suivant: The defensive treaty concluded in the year 1700 between bis late Majesty, King William, of ever-glorious memory and his present Swedish Majesty, King Cbarles XII, etc., $1716 .^{3}$

Du folio io au folio 16 , nous avons le titre ci-après:

The Northern Crisis, or Impartial Reflections on the Policy of the Czar..., London 1716.4

Aux folios I 6-I 8, nous relevons un groupe de trois documents parus de 1726 à 1729 et se rapportant au même sujet:

$A$ second Enquiry into the Reasons of the Conduct of Great Britain, with Relations to the Present State of Affairs in Europe. In a Letter from a Gentl. in the country to his friend in London, London 1726.

An Inquiry into the Reasons of the Conduct of Great Britain with Relation to the present state of Affairs in Europe, London 1727.

$A$ Defence of the Enquiry into the Reasons... (voir plus haut), London 1729.

Aucun de ces textes n'est cité dans les Révélations. ${ }^{5}$

Le document suivant (folios $18-20$ ) est une version allemande du testament apocryphe de Pierre le Grand:

Tagebuch Peters des Großen vom Jabre 1698bis zum Scblusse des Neustädter Friedens, aus dem russischen Original übersetzt, so nacb denen im Arcbive befindlicben und von Seiner Kaiserlichen Majestät eigenbändigen ergänzenden Handscbriften gedruckt worden.

Berlin und Leipzig, $1773 .^{6}$

Les cinq folios suivants sont réservés à des documents et lettres

1 Ici note de Marx: „Blödsinn”.

Ed. E. Aveling, p. 9I sq.

Ibid., p. 60-73.

4 Reproduit dans Secr. Diplom. Hist., p. 27 à 48, mais avec de légères différences. Sur ce document, Marx s'exprime ainsi à Engels: „Eins der auBerordentlichsten Pamphlets, die je geschrieben worden. Hätte mit unwesentlichen modifications im Jahre 1853 erscheinen können. Enthält so wie a. und c. Nachweise der englischen Verräterei."

"Cependant Marx mentionne le second ( 1727 ) dans l'énumération destinée à son ami.

- Marx le considère comme authentique, bien qu'il fût publié après avoir passé par la censure de Catherine II; les faits relatés par les autres documents lui semblent être confirmés par ce testament. 
diplomatiques concernant les rapports anglo-suédois au XVIIIe siècle:

Copies and Extracts of several letters written by the King of Sweden and bis Ministers relating to the Negociations of Baron Görtz etc., published at Copenbagen by order of the King of Denmark. London, 1717 .

Three letters... upon the subject of the Full Power given Bar. Görtz by the King of Sweden... London $1717 . .^{1}$

Sur les deux derniers folios de ce cahier nous avons des extraits de trois documents anglais et français intitulés respectivement:

A Secret History of one Year, London I 714.

Mémoires pour servir l'bistoire de l'empire russe sous le règne de Pierre le Grand, etc. La Haye, i 725 .

Marx en transcrit, par exemple, le passage suivant: «Les Moscovites sont les plus vains et les plus orgueilleux de tous les hommes.... Ce qu'on appelle homme.»

The present State of Russia... 2 vol. (1 722 ).

$$
*_{*}^{*}
$$

Un cahier de 60 folios, daté de février i 8 \% 6 , contient apparemment les documents diplomatiques auxquels Marx fait allusion dans sa lettre à E., à savoir «une série d'autres écrits qui se rapportent occasionnellement à l'histoire anglo-russo-suédoise, ou des brochures anglaises manifestement inspirées par l'ambassadeur suédois Gyllenborg, tels, par exemple, «Remarks on Mr. Jacksons Memorial etc» ". ${ }^{2}$

Sur la couverture intérieure, Marx a noté le sommaire de ce cahier qui comporte vingt-quatre titres.

Le folio I contient quelques lignes tirées des deux documents suivants:

Political conferences between several government men etc., London 1780 . New Dialogues upon the present posture of affairs. London, 1710. Suivent sur trois et demie pages, des extraits de:

The History of Prime Ministers and Favorites in England, London 1763. Les Cours anglaises, de William Ier (1066-1087) à William III (1689-1703), sont passées en revue, avec l'accent mis sur les méthodes ${ }^{1}$ Le premier de ces documents figure sous ,g” dans la liste donnée par Marx à Engels. M. indique ensuite sous „h": Letters which passed between Count Gyllenborg, the Baron Görtz, Sparre, etc. published by authority. London $77 \times 7$. M. remarque ensuite: ,g und h sind natürlich allen Historikern wohlbekannt, es fehlt ihnen aber der Schlüssel zum Verständnis derselben. Beide Publikationen drehn sich besonders um den Plan, den Charles XII, aus Rache gegen England gefaBt hatte, mit einer schwedischen Armee an der englischen Küste zu landen und den Prätendenten zu proklamieren."

Dans Revelations, Marx fait état d'une lettre du Comte Gyllenborg, datée de Londres, 23.I.1717 et adressée au Baron Görtz. Le premier semble s'y faire passer pour l'auteur de "The Northern Crisis", mais Marx le conteste en jugeant d'après ses lettres à Görtz. Revelations, éd. E. Aveling, p. 27.

${ }^{2}$ Classé sous B 80. 
de concussion et de corruption pratiquées par les grands hommes politiques pour gagner les parlementaires. A propos du dernier, retenons ce passage noté par Marx: "The constant advice of his pretended friends and ministers to him was: «Borrow what you can; the more you borrow the more friends you make; interest is a stronger tie than principle.)"

Memoirs of the Conduct of ber late Majesty and ber last Ministry relating to the Separate Peace with Tories. By the Right Hon. the Countess of [?], London 1713.

An Inquiry into the Miscarryings of the 4 last years etc. London 17 I 4. Marx en transcrit quelques lignes, puis, sur les folios 4 à 9, accumule les extraits d'un document allemand dont voici le titre:

Urkunden und Materialien zur näberen Kenntnis der Gescbicbte u. Staatsverwaltung nordischer Reiche [Gaspary?], I 786-1 790, 3 vol.

Les documents dépouillés (en allemand et en français) évoquent les rapports, dans la première moitié du XVIIIe siècle, entre le Danemark et la Suède, le traité entre la Grande-Bretagne, la France et le Danemark du 16 avril 1727 , les conquêtes nordiques de la Russie, les conflits russo-turcs, etc.

William (J.), The Rise, Progress and Present State of Nortbern Governments, 2 vol. 1747 .

Deux pages d'extraits (folios 9 et 10); ce livre est repris plus loin dans le même cahier (folios 26 à 30). Récit du séjour de Pierre le Grand en Hollande, sa rencontre avec William III, toi d'Angleterre, son apprentissage comme constructeur naval, ses guerres avec la Suède (avec l'appui de l'Angleterre!), etc.

Nous arrivons maintenant à un lot de documents dans lequel Marx a beaucoup puisé pour ses Révélations. Il s'en exprime comme suit dans sa lettre à Engels, du 29.2.1856: «Im Museum habe ich fünf Foliobände Manuskripte über Rußland (nur über das achtzehnte Jahrhundert) aufgetrieben und ausgezogen. Sie gehören zum Nachlaß des Archdeacon Coxe, der wegen seines Sammlerfleißes bekannt ist. Enthalten viele Originalbriefe (bisher ungedruckt) der englischen $\mathrm{Ge}$ sandten in Petersburg an das hiesige Kabinett, worunter machen very compromising indeed. Aus dem Jahre 1768 , befindet sich ein Manuskript darunter von einem der Gesandschaftsattachés über (den Charakter der russischen Nation> ".

Le cahier que nous examinons donne, en effet, ces documents aux folios I I à 24, sous le titre et la cote du British Museum, que Marx a notés comme suit: «Coxe Papers (vol. I 81 ) $N 9258$ ".

Suivent les documents suivants:

Papers relating to Denmark and Sweden, enthält u.a. Geschichte d. Krakehls zw. Gustav III u. s. Mutter... 
Papers relative to Russia (vol. 178-181). Vol. 178 :1 Tableau topographique de l'Europe de Russie; 2 Curious Paper on the Genious and Character of the Russians...

Voici quelques passages notés par Marx de ce papier autographe datant apparemment de 1762 : «[...] such particuliar marks and striking features there are surely to be found in this action, notwithstanding all the pains that have been taken to bend or distort them to the likeness of others. The common people, though not laborious are strong and handy, patiently bearing the extremes of heat, cold and hunger to an astonishing degree, yet in general they are lazy, indolent and sensuel. Knowing an happiness beyond the gratification of drunkenness and gluttony; they are hospitable, charitable and good natured, [...] they are humane, and by no means justly accused of cruelty [...]. All the affections of the soul seem weaker in them, than in most other nations. They are therefore formed to be commanded.»

Parlant des nobles:

«[...] either so depraved are their dispositions, or so perverted their judgements, that they derive few advantages from birth or education which claim to respect of others, or are of use to themselves [...] French adventurers received into most families in open arms, in quality of secretaries, librarians, readers, preceptors or parasites [...]

They are vain, high, and many of them interested, [...] bold and adventurous in the pursuit of pleasure, $[\ldots]$ people barbarous, $[\ldots]$ ignorant, nobility, but half civilised [...] to despotism Russia ows her greatness and dominion, so that if ever the monarchy becomes more limited, she will lose her power and strength in proportion as she advances in moral virtue and civil improvement.»

$\mathrm{Au}$ folio 14, nous tombons sur le document qui forme la première pièce à conviction publiée par Marx dans ses Révélations: la lettre du diplomate anglais Rondeau à Horace Walpole, Pétersbourg, 17 août I 736 , au sujet de ses efforts pour décider le tsar à conclure la paix avec les Turcs. ${ }^{1}$

Au folio I 5 , nous lisons:

Dispatch from Mr. Finch to lord Harrington, St. Petersburg, nov. 1740 (in the Revolution that had happened in Russia). Note de Marx: «Der Esel Finch gratuliert sich daß er, in Gegensatz zum frz., gr., schwed., oester. Gesandten, sofort d. Barons s. cour macht, an die Einzigkeit seiner Regierung (?) glaubt. Er hat I. März zu berichten in d. nächsten Brief:

Id. to id. Petersb. I 8.1 I.1740, [...] statt von seiner eignen (Blindheit) spricht Finch v.d. sstrange fatality and blindness of the Prince of Kurland,."

${ }^{1}$ Secret. Dipl. Hist., p. 7 sq.; B. Hepner, o.c. p. 94. 
Après quoi, nous avons (folios Is à I 8):

Sir George Macartney to the Earl of Sandwich. St. Petersburg Ist (I 2th) March 1765. Most Secret.

Cette lettre constitue la pièce $\mathrm{n}^{0} 2$ reproduite par Marx dans ses Révélations. ${ }^{1}$

Le document suivant est reproduit dans les Révélations comme pièce $\mathrm{n}^{0} 3$ :

Sir James Harris to Lord Grantham. 16 (27) Aug. 1782 (Private). ${ }^{2}$ Marx mentionne cette lettre avec celle de Rondeau et de Macartney dans les Révélations comme exemples de la bêtise des diplomates anglais traitant avec la Russie. ${ }^{3}$

Nous trouvons en outre:

Mémoire sur le Commerce en Russie. Mons. le Procureur Général, le 26 juin I76r.

Document officiel dont Marx note quelques remarques sur les activités commerciales en Russie, le commerce de luxe, les privilèges, les productions, etc. C'est peut-être une des sources utilisées par Marx pour rédiger le chapitre III de ses Révélations où il aborde la question des rapports commerciaux anglo-suédo-russes, ${ }^{4}$ sur lesquels on trouve plus loin d'autres ouvrages consultés.

$$
\text { ** }
$$

Outre les documents que nous venons de passer en revue et dont nous avons retrouvé la trace soit dans des publications de Marx, soit dans sa correspondance, le cahier portant la date de 41856 » nous apprend que l'infatigable lecteur a consulté un des plus célèbres fonds de manuscrits du British Museum: la Sloane Collection. Sous les cotes 4903, 4207, 4459, 4107, 4224, 4193, Marx a retrouvé une série de documents qui le renforcent sans doute dans son désir de pénétrer encore plus intimement dans les arcanes tortueuses de la diplomatie secrète. Cela explique sans doute le passage de l'Adresse inaugurale de 1864, dans laquelle Marx exhorte les ouvriers à se familiariser avec la diplomatie secrète de leurs gouvernements.

Il s'agit de documents qui illustrent l'histoire politique et diplomatique de l'Europe du i 8 e siècle. Ils sont pour la plupart rédigés en français et en allemand et Marx en transcrit (ou traduit) des passages (en allemand).

Voici quelques titres relevés sur les folios 37 à 52 .

Georg v. Hanover an die Queen Anne, I9 Febr. 1706.

Histoire politique du siècle depuis $164^{8}$ jusqu'à 1788 . Par l'éditeur du

1 Secret Diplom. Hist., p. 8-1 r. B. Hepner, o.c., p. 94-99.

2 Secret Diplom. Hist., p. 12-19. B. Hepner, o.c., p. 100-1 I3.

${ }^{3}$ Secret Diplom. Hist., p. 22 sq. B. Hepner, o.c., p. 120 sq.

4 Secret Diplom. Hist., p. 5 I sq. B. Hepner, o.c., p. I 66 sq. 
Testament politique du Card. Alberoni (...) Lausanne ro oct. 1753. Parmi les faits et dates relatés, nous retenons le traité des Pyrénées de I660, le traité d'Oliva entre les puissances du Nord, la Triple Alliance Angleterre-Hollande-Suède (I668), le traité d'Aix-la-Chapelle (I668), le traité secret entre Louis XIV et Charles II (1670), les traités de Nimègue (1678-79), Utrecht, Rastadt (I 7 13-I 4), la Triple et Quadruple Alliance (172I), la paix de Tramendahl (Is avril i 700), le traité de Passarovitz (1715), l'alliance défensive entre la Suède et la Turquie, l'alliance russo-britannique. Marx note ce passage: «La cour de St Pétersbourg (unter d. Elisabeth) ne manquait point d'affirmer aux Finlandais, avant que de les attaquer, de les mettre en République indépendante pour étre désormais sous sa protection une barrière entre les deux empires [...]". ${ }^{1}$ Paix d'Aix (1748). Diverses lettres du plénipotentiaire anglais James Jefferys, datées I 7 I I, copie de la lettre de Mons. de Stanyan à M. le Général de St. Saphorin, écrite de Constantinople, 22 juillet 1719 , sur les rapports russo-turcs. Retenons cette phrase: «Car quoi qu'on en dise, je vois clairement que les Turcs ont plus confiance aux Français qu'aux autres nations chrétiennes. Mais il faut surtout prendre garde que l'on ne s'aperçoive pas que cet avertissement vient de moim.2

Instruction pour $M$. Witworth allant à Berlin, mars 17 I9.

Plan d'un traité avec $S a M$. Danoise, mars I7I9.

Aux folios 47 à 50 , nombreux extraits d'un document anglais sous le titre "Character of King William (The Dutchman)", après quoi aux folios so à 53: Projet d'alliance entre le roi d'Angleterre, comme électeur de Brunswick et le roi de Prusse ( "la mort de S. M. suédoise devait naturellement faciliter le rétablissment de la paix du Nord»). Plan du comte de la Marche pour la paix du Nord.

Les derniers folios ( 56 à 58 ) donnent la suite des Sloane Papers, avec les mêmes thèmes que les précédents: plan projeté pour la Paix générale du Nord à Brunswick; correspondance entre Frédéric Guillaume II et George Irr; considérations sur le traité entre l'Angleterre et la France; extrait de ce traité.

Alors que Marx collectait à Londres les documents pour l'oeuvre projetée sur l'histoire de la diplomatie secrète, Engels travaillait à Manchester sur un problème auquel les deux amis attachaient beaucoup d'importance politique: le panslavisme. ${ }^{3}$ Tout en poursuivant ses recherches, Marx rassemblait des matériaux pour l'oeuvre de son ami.

1 Folio 41 .

2 Folio 45.

${ }^{8}$ Il avait déjà donné deux articles à la Neue Oder-Zeitung, 2 I et 24 avril I 8 ss. Réimprimés dans GS II, p. 227-233. 
On peut s'en rendre compte d'après leurs lettres échangées au cours de février et mars 18,6 .

Les folios 24 à 25 , puis 30 à 36 et 53 à 55 , nous donnent principalement la liste des Slavica, notée à l'intention d'Engels : ${ }^{1}$

Dobrowsky's Slavin. Botschaft aus Böhmen an alle slavische Völker etc. $2^{\text {te }}$ verb. Ausgabe von Wenceslaw Hanka. (...) Prag I 834.

On trouvera une description critique et le sommaire détaillé de ce recueil dans la lettre adressée par Marx à Engels, le 29 février $1856 .{ }^{2}$

Das Slaventum v. Moritz Wilhelm Heffter, Leipzig 1852.

Les extraits concernent l'histoire et la préhistoire des slaves, leur langue, leur répartition géographique. ${ }^{3}$

Eichhoff F. G., Histoire de la langue et de la littérature des Slaves etc. Paris 1839.4

Les extraits, peu nombreux, se rapportent à l'épopée d'Igor et à la poésie nationale des divers peuples slaves; pour la littérature russe, ils s'arrêtent à Pouchkine.

L'aperçu que Marx donne de cet ouvrage dans sa lettre du s mars, nous montre qu'il ne se bornait pas seulement à lire et à transcrire, mais qu'il étudiait ses matériaux avec un esprit critique lorsqu'il s'agissait d'un problème sur lequel le "parti Marx» avait à prendre position devant le public.

$$
\text { ** }
$$

Après cet intermède slave auquel Marx se livrait pour documenter Engels, nous retrouvons, dans un cahier daté d'avril 1856 , une série de documents diplomatiques dont certains figurent déjà au cahier

1 Presque tous les titres communiqués par Marx à son ami dans ses lettres du 29 février et 5 mars 1856 se retrouvent dans le cahier que nous examinons.

2 En voici un passage: „Dobrowsky schreibt in einem knotenhaft naiven Stil, mit der höchsten Kordialität gegen seine 'seligen' oder noch lebenden deutschen Kollegen. Das einzige, was mir interessant in der 'Slavin' schien, sind die paar Stellen, worin er die Deutschen direkt als die Väter der slavischen Geschichts- und Sprachforschung anerkennt."

Sur cet opuscule, voir M. à E. 29.2.1856. Du même auteur, Marx note en outre un titre que nous n'avons pas rencontré dans ce cahier: Der Weltkampf der Deutschen und Slaven seit dem Ende des fünften Jahrhunderts, 1847 . „Der Verfasser gesteht in der Vorrede selbst, daB er eigentlich nur genau und originell bewandert ist in der slavischen Geschichte soweit sie das preußische 'Vaterland' betrifft. Von den 48 I Seiten des Buches werden über drei Viertel eingenommen von der Periode vom Ende des fünften Jahrhunderts bis I 147. Der Rest geht nur gelegentlich und ganz kursorisch über das dreizehnte oder gar vierzehnte Jahrhundert hinaus."

4 Dans sa lettre du 5 mars, Marx donne à Engels un aperçu intéressant concernant sa lecture et les idées qu'il en tire et nous trouvons là une sorte de modèle de collaboration entre eux.

Dans la même lettre, on trouvera d'autres titres fournis par Marx à Engels à propos de la poésie et de la musique des peuples slaves, des migrations des slaves occidentaux, les Slaves hongrois, les Serbes, etc. 
précédent et que Marx reprend pour compléter son information sur la «trahison anglaise ${ }^{1}$

Les Sloane Papers y fournissent (folios I à 6) de nouveaux extraits, toujours sur la Russie. La première pièce du dossier porte le titre:

Office Papers in French concerning the title given to the Czars of Moscovy. ${ }^{2}$

Marx s'intéresse, avec une minutie dont les Révélations nous donnent l'explication, aux titres des Czars de Moscovie: il y a vu la preuve de la «servilité abjecte» et de la «soumission cynique» des hommes d'Etat anglais à l'égard de la Russie. ${ }^{3}$

En voici un échantillon recopié par Marx:

«La coutume [...] a toujours été d'écrire aux Czars de Moscovie sur du velin enluminé peint et doré, comme on fait aux Empereurs de Maroc et de $\mathrm{Fez}$, et à plusieurs autres Princes non européens, lesquels selon cette coutume seraient également fondés d'insister sur le Titre d'Empereur. On n'a jamais voulu changer l'usage étably quoique les moscovites l'ayent fort sollicité - durant l'Ambassade de Mylord Wbitenorth à Moscou. Ce ministre s'excuse toujours d'en faire la proposition. Il leur dit qu'il leur donnerait le Titre, sans difficulté, tel qu'il le trouvait étably; mais qu'il ne leur conseillait pas de remuer cette matière, ni de s'éclairer trop soigneusement sur quel pied on leur donnait ce titre. Les Moscovites crurent son avis bon pour lors.»

Dans la même collection, un document anonyme se rapportant à l'Espagne et l'Angleterre, la Quadruple Alliance, la Prusse et l'Italie; la France, médiatrice dans la guerre du Nord, etc. ${ }^{4}$

Dans le même souci de vérifier ce qui a toujours été à ses yeux la politique de l'Angleterre vis-à-vis de la Russie, Marx a fait des lectures fouillées de:

Rev. T. S. Hughes, The History of England from the accession of George III. I $760-1835$. 7 volumes, London 1836 .

Les extraits et notes sur les folios 7 à 14 tournent autour d'un seul sujet: l'attitude russophile de certains hommes d'Etat anglais au moment où la Russie était en guerre avec la Turquie et où son conflit avec la Suède ne cessait de s'aggraver (1773); le différend qu'opposa, lors de la guerre coloniale, lord Sandwich à Fox, Burke, Pitt (17781779).

L'ensemble de ces notes a été utilisé par Marx pour les commen-

1 M. à E., 12.2.1856. Le cahier est classé actuellement sous B 73 .

2 Enregistrée au catalogue sous la cote 4 I93, no 3 I.

${ }^{3}$ Secret Diplom. Hist., p. 22. B. Hepner, o.c., p. I19. „England war auch die erste europäische Großmacht, die seinen (il s'agit de Pierre le Grand) Kaisertitel anerkannte, etc." M. aे E., I 2.2.1856.

4 No 32. 
taires qu'il écrivit aux documents $n^{\circ s} 2$ et 3 publiés dans le Chapitre Ier de ses Révélations. ${ }^{1}$

De même caractère sont les extraits de:

Lord Mabon, History of England from the Peace of Utrecht to the peace of Aix-la-Chapelle, $2^{\mathrm{d}}$ ed. London, 1839.

Après une notation sur les sources inédites complétées par l'auteur, Marx enregistre avec soin l'ascension de la Russie, sous Pierre le Grand, au rang de grande puissance européenne, la Suède étant déchue de sa position de force dans le Nord de l'Europe. Et c'est de nouveau l'examen, à partir de nouveaux documents autographes, de la politique des whigs sous George I ${ }^{\text {er }}$ - des Stanhope, Walpole, Townshend, etc. dont Marx s'obstine à vouloir démasquer le jeu prorusse.

La chronologie détaillée dont ces pages sont parsemées semble témoigner chez Marx du désir de démêler soigneusement la trame du tissu politique qu'offraient, pendant la guerre nordique et après la mort de Charles XII, les relations entre les diverses puissances européennes jusqu'à la paix de Nystadt (1 72I). L'oeuvre de Schlosser, History of the 18 th Century, est copieusement exploitée (folios 32 à 42) et on peut penser que Marx tenait en haute estime cet historien dont il lira plus tard la Weltgescbichte avec un zèle dévorant. ${ }^{2}$

Nous avons ensuite de nouveaux passages (folios 43-44) extraits de Truth is but Truth..., déjà rencontré dans un cahier précédent. Ils sont suivis (folios 44-45) de la pétition de marchands anglais au parlement citée par Marx au chapitre III de ses Révélations: The case of the merchants trading to Russia. ${ }^{3}$ Du même ordre est le document intitulé: Further reasons for enlarging the trade to Russia bumbly offred by the Merchants and Planters trading to, and interested in the plantations of Virginia and Maryland.

C'est à ces réclamations antisuédoises de ces marchands que Marx oppose les chiffres qui prouvent que pendant la première partie du XVIIIe siècle le commerce anglo-russe ne représentait qu'une fraction insignifiante du commerce global de l'Angleterre. ${ }^{4}$

De nouveaux extraits du pamphlet intitulé The Northern Crisis, largement reproduit dans les Révélations, figurent aux folios 44-46 de ce cahier. ${ }^{5}$ De même le problème de la flotte du Czar dans la Baltique, traité par Marx, dans le même écrit, se repose avec les titres suivants: Reasons for the present conduct in relation to the trade in the Baltick, set

${ }^{1}$ Secret Diplom. Hist., p. I I sq. note 9 et p. Is sq. notes I 2 à 17. Hepner, o.c., p. 99 sq.

- Voir BIBL. 75 I p.

- Secret Diplom. Hist., p. 54. B. Hepner, o.c., p. 171 .

I Ibid., p. so sq. et 16 I sq.

- Les passages copiés se retrouvent dans Secret Diplom. Hist., p. 32 sq. 
forth in a letter of a gentleman at Dantzick, to bis friend at Amsterdam, Translated from the French (...) London 1715.

An account of Russia as it was in the year 1710 . By Charles Lord Withworth, 1758 (Strawberry-Hill).

Mémoire présenté à Sa Majesté britannique (par un ministre du Czar) 1717 .

Nous retrouvons la chronologie (I 70I-I732) relative à la Triple et Quadruple Alliance, aux tractations entre l'Angleterre et la Hollande, aux contacts avec la Russie, avec le vol. I de Charles-Louis de Sevelinges, Mémoires secrets et correspondance inédite du cardinal Dubois..., 2 vol. Paris i 8I g. C'est l'alliance contre le Czar proposée par Charles XII à la Porte (17IO-I 7 I 2) qu'évoque W. Theylls (...), Mémoires pour servir à l'bistoire de Charles XII etc. Leyde I 722.

De la chronologie, encore et toujours, avec Ralph vol. II: The History of England etc. 1796, Le Czar et la Suède, 1700.

Anderson (Adam) An bistorical and cbronological deduction of the Origin of Commerce, 2e éd. 4 vol. (1 787-1 789). De ce travail, Marx extrait des chiffres sur le Commerce de la Baltique que l'on retrouve dans les Révélations. ${ }^{1}$

Avec le cahier daté de mai i 8,6 , nous passons du domaine politique à la philologie. ${ }^{2}$ Marx souffrait alors de fortes douleurs rhumatismales et était incapable d'un travail de recherche méthodique. ${ }^{3} \mathrm{C}$ 'est, semblet-il, pour se distraire qu'il lut Hermann Grimm, Geschicbte der deutscben Sprache (1853).

Ce cahier offre tous les caractères d'un bon travail d'étudiant germaniste avide d'assimiler les règles et lois de transformation qui ont présidé à la formation de l'allemand moderne: étymologie, tables des vocalismes, grammaire comparée, déclinaisons, etc. On est en présence d'un abrégé composé avec un soin méticuleux, et on croirait que l'auteur se préparait pour un examen de philologie. Nous avons là un témoignage du goût que Marx avait pour l'histoire des langues, mais aussi de ses préoccupations méthodologiques: les explications étymologiques ne sont pas absentes de ses travaux scientifiques, et Marx tenait Grimm en haute estime.

$$
\text { ** }
$$

Le cahier non folioté daté d'août 1856 nous ramène aux études historiques proprement dites et fait partie du groupe des cahiers «diplomatiques" de Marx. ${ }^{4}$ Il s'ouvre sur une impressionnante liste de

1 Secret Diplom. Hist., p. 5 I sq.

2 Classé actuellement sous B 78 .

${ }^{3}$ Cf. M. à E., 23. 5. et 5.6 .1896 .

4 Il est possible que Marx se soit trompé d'année. Il empruntait parfois les cahiers d'école 
documents politiques figurant dans les collections manuscrites conservées au British Museum. Pour certaines, nous relevons l'indication suivante:

«(7058-7079) (22 vol.) The Correspondence of Engl. ministers either at home or abroad with George Stepney, Esq. to several of the Europe Sates betw. the years 1690 and $1707 \%$. Il s'agit très vraisemblablement des Sloane Papers, déjà mentionnés dans le cahier daté d'avtil 1856 qui contient également le début des extraits des Mémoires de W. Theylls, dont nous retrouvons ici la suite sur six pages. Ce sont les événements postérieurs à la paix du Prut ( $17 \mathrm{I}$ I), épisode de la guerre du Nord: Charles XII, allié des Turcs, en devient le prisonnier à Bender (I 713 ) et ne rentrera en Suède qu'après l'équipée de Stralsund (I 7 I ). Parmi les documents recopiés par Marx, mentionnons plusieurs lettres du sultan à Israël Costa, gouverneur de Bender (1 712 2, 1713 ).

Au même thème se rapporte une partie des passages tirés des dix volumes de A. L. Schlözer, Briefwechsel [...] bist. u. polit. Inbalts ze éd. Göttingen (1776-1 782). ${ }^{1}$ C'est l'histoire de la grande puissance nordique sous Charles XI, Charles XII et Charles XIII. Les notations sont interrompues pour faire place à des passages tirés de: Dr Luthers Handelspolitik (Aus den Büchern von Kaufhandel u. Wucher v.J. I 824). ${ }^{2}$

Du riche recueil de Schlözer proviennent sans doute les quatre pages que Marx a remplies avec des extraits des Anecdotes du séjour du Roi de Suéde à Bade, publiées par le Baron de Fabrice (Hambourg 1760 ). Les lettres de ce dernier transcrites par Marx concernent l'épisode turco-suédois de la guerre du Nord (I710-1713). Une citation de l'Histoire de Charles XII de Voltaire se rapporte à la même époque, notamment l'annexion des pays baltes par Pierre le Grand (folio I9). Avec les extraits des Mémoires pour servir à l'Histoire du XVIIIe siècle de De Lamberty (1724 et suiv.), nous remontons aux origines de la guerre du Nord (entente entre Pierre le Grand et Auguste II, roi de Pologne) et à la tradition anglaise. ${ }^{3}$

Nous trouvons en outre dans ce cahier des traces nombreuses de divers travaux et documents historiques que Marx avait utilisés en

à une de ses filles et on lit sur celui-ci: „Miss Marx April 7th I854”. En mai 1854, Marx souffrait d'un furoncle au visage, ses enfants avaient la rougeole si bien qu'il pouvait tout au plus „se distraire”. - Actuellement classé sous B 75 .

1 Marx en cite un passage au chap. $V$ de ses Révélations.

Marx en cite toute une page en rédigeant le livre II du Capital. Voir ibid., section IV, chap. 20 ; note 49.

Voir Secret Diplom. Hist., p. 5o sq. B. Hepner, o.c., p. 165. sq. Aux folios 26, 30 à 33 , ss à 59 de ce cahier nous avons sous le titre The political State of Great Britain, des détails sur les rapports commerciaux anglo-suédois (dont les Révélations font état au chap. III) et sur les relations anglo-russes en 1719 . 
partie et qu'il voulait sans doute exploiter pour la suite qu'il pensait donner à la série d'articles publiée dans The Free Press à titre d'Introduction:

Motley, The History of the Life of Peter I. London 1739. 3 vol. (folios 27 à 29).

Historical Notes by F. S. Thomas. London 1856 (folios 40-4I).

Kerble, Statepapers and Correspondance... London 1857.

Sir George Murray, The letters and dispatches of Jobn Churchill, first Duke of Marlborough from $1702-1712$. London I 845.

Cours d'bistoire des états européens depuis le bouleversement de l'Empire Romain d'Occident jusqu'en 1789 par Max. J. F. Schoell. Paris I 830-1834 (folios 44 à 48). Les extraits proviennent des volumes 9 et 10 .

J. J. Schwartz, Einleitung in die Staatswissenschaft, 1744 (folios 48 à 55 ).

Toutes ces sources sont centrées sur l'implantation de la puissance russe en Europe sous Pierre le Grand.

Cependant, à partir du folio 34 et jusqu'au folio 40 , nous tombons sur des annotations qui nous font passer de l'histoire diplomatique du XVIIIle siècle à un événement plus récent: la guerre anglo-afghane de 1842 . En effet, Marx a dépouillé dans la livraison de février i 843 de l'Edinburgh Revien une étude intitulée Ministerial Misrepresentation regarding the East. C'est l'analyse de la campagne de Caboul (1842) «which ended in the retreat and destruction of the British Army».

Les notes de Marx retiennent particulièrement les phases de la politique palmerstonienne dans cette partie de l'Asie depuis 1833 jusqu'à la rupture, en I 838 , entre l'Angleterre et la Perse. Sans doute préparait-il alors la matière des articles qu'il donna à partir de janvier I 857 à la NYT sur le nouveau conflit anglo-persan. ${ }^{1}$ Pour Marx, il s'agissait surtout de découvrir derrière le prétendu libéralisme de la politique extérieure de Palmerston, les concessions réelles faites à l'expansion russe en Extrême-Orient. ${ }^{2}$

Il faudrait ici mentionner les pages d'un cahier précédemment examiné, non daté, et dont nous avons dit que son contenu correspondait à plusieurs moments des études de Marx. ${ }^{3}$

Il s'agit de deux pages non foliotées qui font suite aux notes prises par Marx à propos de certaines théories de la monnaie et du crédit, donc à un moment où, provisoirement certes, il reprenait son oeuvre scientifique, parce qu'il s'attendait à l'imminence d'une crise financière aux dimensions européennes. ${ }^{4}$ C'est le brouillon, plein de ratures,

${ }^{1}$ Cf. BIBL. 460, 473, 476. Voir M. à E., 30.10.1856 et 23.1.1857. Engels assuma la partie militaire des correspondances envoyées par Marx à la NYT.

${ }^{2}$ Voir M. à E., 18.3.1857.

${ }^{3}$ Cahier classé sous B 79.

4 Voir CHR., p. 160. M. à E., 26.9.1856. 
d'un article destiné à la NYT, intitulé The War against Persia. Marx s'efforce de «démasquer» Palmerston qu'il accuse de reprendre en I8 66 le rôle douteux qu'il avait joué près de trente ans auparavant lors des conquêtes russes en Perse.

$$
\text { ** }
$$

Un cahier dont nous ignorons la date semble se situer dans la même periode que le précédent, du moins par les extraits notés sur la Pologne, par certaines statistiques relatives au budget anglais, par des allusions aux incidents de Chine. ${ }^{1}$

Jusqu'à la quinzième page, ce cahier ne comporte pas de foliotage. Il comprend, dans cette partie et sur cinq folios, des extraits et des résumés en allemand de J. Lelewel, Histoire de la Pologne. Paris r 847. C'est surtout une chronologie, sur trois colonnes, des grandes périodes historiques de la Pologne de 280 à 1795 . Suivent, sur plusieurs pages, des notes économiques et une sorte de brouillon d'article intitulé "The Budget of Sir G. Lewis" où on lit des remarques sur le conflit anglo-chinois en $\times 857$, les massacres de Canton, la défaite parlementaire de Palmerston devant la coalition Disraeli-Gladstone-Russell.

Une lacune de quatre pages nous prive du début et donc du titre d'une longue série de notes historiques, en français, en allemand et en anglais, dont il est cependant facile d'établir la provenance. Il s'agit en effet de résumés de lecture d'après l'ouvrage d'Elias Regnault, Histoire politique et sociale des principautés danubiennes paru à Paris, en $18550^{2}$ Extérieurement ces pages se distinguent des précédentes à la fois par l'écriture et par l'encre employée, donnant l'impression d'avoir été remplies à un tout autre moment, vraisemblablement quelques mois auparavant. En fait, dès le début de 1856 , Marx a envoyé à la NYT plusieurs articles sur les principautés danubiennes. ${ }^{3}$

Nous avons à faire ici à un récit succinct du destin de la Moldavie et de la Valachie, du dernier tiers du XVIIIe siècle au traité turcoautrichien de 1854 , et de la retraite des troupes russes consécutive à cet acte de garantie en faveur des principautés roumaines.

Marx termine ce tableau historique par ces phrases en anglais: «Thus we arrive at the same conclusion which we exposed at the outbreak

${ }^{1}$ Classé actuellement sous B 85 . Voir BIBL. 464 et 465 . Dans la lettre adressée d̀ Engels le 2.12.18 56 , Marx parle de ses „récentes études sur l'histoire polonaise”, qui l'ont incité à prendre "décidément" parti pour la Pologne.

Marx le cite élogieusement dans ses Révélations. Voir Secret Diplom. Hist., chap. III, p. 56.

Un seul en parut à la date du 23 janvier 1856 comme éditorial. Les autres furent renvoyés, la rédaction de la NYT se trouvant alors sous l'influence du panslaviste Gourovski qui s'érigea en censeur des manuscrits de Marx. Voir M. à E., 10.10.1856 et 6.2.1857. 
of the present war. ${ }^{1}$ The questions involved in the so called oriental question must be solved in the Russia sense, if Europe maintains her present constitution or they must altogether assume[? present?] a new aspect. It becomes identical[?] with the cause of a general European Revolution. The present status quo, founded on the mere independance and integrity of the Turkish Empire may be artificially protracted. It can never afford anything else but momentaneous shifting of the difficulties and mock solution."

\section{CONCLUSION}

De 1852 à 18,6, Marx a fait principalement métier de journaliste rétribué à la page. Il écrivait beaucoup, mais sans enthousiasme, parfois sans conviction, le plus souvent avec répugnance. ${ }^{2}$ Mais il trouve une compensation, sinon une justification morale, dans les études historiques auxquelles les sujets d'actualité l'invitent, et s'estime heureux d'être "payé à étudier» ${ }^{3}$ Ces cahiers de lecture remplis pendant cette période de près de cinq années, tout comme sa correspondance avec Engels, témoignent de la satisfaction qu'il éprouve à lire et à copier, à traduire et à compulser. Ainsi le journaliste dont il doit assumer le rôle pour subsister matériellement, fait place à l'historien et au sociologue, qui a le goût et la passion de la culture historique. L'événement du jour n'est dès lors que prétexte à recherches, et bientôt l'occasion de découvertes qui permettent de serrer la vérité historique. A la lecture des textes imprimés vient s'ajouter le dépouillement de documents inédits dont le British Museum possède les collections les plus riches. Et bientôt l'irrépressible curiosité du lecteur et du chercheur se changera en besoin de création littéraire, en volonté de faire oeuvre d'historien, - mais aussi et surtout de critique social. Il ne faudrait cependant pas, redisons-le, sous-estimer les travaux journalistiques de Marx. S'il n'y peut donner toute sa mesure, si son esprit critique ne peut s'y manifester pleinement, s'il est obligé de compter avec une rédaction et un public peu préparés pour saisir des vues théoriques et des idées révolutionnaires, en un mot s'il doit sacrifier au goût moyen et médiocre de l'information rapide, il réussit souvent à insérer dans ses correspondances des pages et des passages

1 Il s'agit évidemment de la guerre de Crimée commencée en mars I854 et terminée deux ans plus tard par le traité de Paris.

2 „Es ist in der Tat ekelhaft, da $B$ man verdammt ist, es als ein Glück zu betrachten, wenn ein solches Löschpapier einen mit in sein Boot aufnimmt. Knochen stampfen, mahlen und Suppe draus kochen wie die Paupers im Workhaus, darauf reduziert sich die politische Arbeit, zu der man rechtlich in solchen concern verdammt ist," M. à E., 23.I. 1857 .

${ }^{3}$ A E. 2.9 .1854 
qui trahissent une réflexion fondamentale et une conviction d'homme de science.

Ces pages, le lecteur attentif les découvrira sans peine dans tout ce que Marx a écrit sur la question d'Orient, les révolutions espagnoles et la diplomatie secrète: trois thèmes qui forment le contenu principal des articles publiés par Marx au cours de la période considérée. Or, il est évident que les matériaux déposés dans les cahiers d'étude dépassent de loin, par leur nombre et leur importance, le simple canevas de quelques correspondances de presse. S'ils ont servi à cette fin seulement, c'est que les circonstances n'étaient pas favorables à un travail historique au sens vrai du terme. Pourtant le souci de la vérité historique est presque toujours présent dans ce que Marx écrit pour la presse, ne serait-ce que par l'évocation de l'arrière-plan éloigné auquel le narrateur s'efforce de relier l'événement actuel. Tel est le cas de tout ce qu'il a écrit sur la question d'Orient et sur les révolutions espagnoles. Pour le premier sujet, l'actualité (guerre russo-turque, guerre de Crimée) se trouve éclairée par des coups de sonde dans le passé, expliquée par le rappel des circonstances qui ont conduit la Russie à convoiter l'héritage de «l'homme malade», et la France et l'Angleterre à se contenter d'un simulacre de victoire. Dans ses cahiers d'étude, Marx se borne le plus souvent à enregistrer et à classer les faits et les gestes, à exprimer les silhouettes du dossier qu'il veut ouvrir. Bien sûr, la part de "l'instinct historique» n'est pas négligeable dans l'utilisation que Marx fait parfois de cette matière brute, par exemple pour démontrer la duplicité du jeu politique de Palmerston, définitivement jugé comme serviteur des intérêts russes. Sous la plume d'un tel polémiste, la monographie historique devient facilement une miniature littéraire: tels les portraits de «Pam» et de Russell.

On pourrait en dire autant des séries d'articles que Marx a consacrés à l'histoire des révolutions espagnoles. Obligé de rapporter sur les événements de 1856 , il estime indispensable de revenir à 1812 et 1820 , d'étudier l'institution des juntas et des cortes, de scruter le caractère national de l'Espagnol, sa langue, ses moeurs, sa religion, ses attitudes. Et lorsqu'il choisit Espartero comme type représentatif du "caractère» espagnol, la description trahit en quelque sorte la volupté avec laquelle il accumule ses notes d'études, apprend l'espagnol et se penche sur les créations institutionnelles qui ont fait l'originalité de l'Espagne révolutionnaire.

En commençant ses recherches dans le domaine de l'histoire de la diplomatie, Marx semble être pris par l'ambition de découvrir une vérité historique dont il estime qu'elle avait échappé aux historiens de métier. Sans en être conscient, il se laisse guider dans ce travail par sa passion politique: il faut que la «russophilie» de Palmerston obéisse 
à une ancienne tradition whig dont les origines remontent au début du XVII siècle, notamment à l'époque de la guerre nordique. L'importance que Marx attribue à cette enquête semble être démesurée: surtout si l'on sait qu'il considérait les articles qu'il donna à l'organe du russophobe Urquhart comme une simple aintroduction" à une oeuvre fondamentale. ${ }^{1}$ Cet acharnement à dépister les complicités anglaises dans l'ascension de la Russie au rang de grande puissance européenne est caractéristique de l'attitude que Marx n'a cessé de prendre, en tant qu'homme de parti et révolutionnaire, contre la politique des Tsars vis-à-vis de la Turquie, de la Pologne ou de l'Extrême-Orient.

A partir de la fin de 18,6 , Marx trouvera de nouveau le loisir de reprendre son oeuvre économique; il délaissera alors les lectures historiques, du moins pendant une assez longue période. Mais on retrouvera jusque dans son oeuvre maîtresse (dont la première tentative de rédaction date de $1857^{-1858)}$ les traces des études auxquelles ses activités journalistiques l'ont conduit.

1 Engels encourage fortement son ami de persister dans son dessein. Voir la lettre de Marx à sa femme, datée du 21 juin 1856 in Annali, Milan, I, 1958, p. 154. 